\title{
Benchmarking of Data Fusion Algorithms in Support of Earth Observation Based Antarctic Wildlife Monitoring
}

\author{
Chandi Witharana $^{1 *}$, Michelle A. LaRue ${ }^{2}$, Heather J. Lynch ${ }^{1}$ \\ ${ }^{l}$ Dept. of Ecology and Evolution, Stony Brook University, Stony Brook, New York, USA \\ ${ }^{2}$ Dept. Earth Sciences, University of Minnesota, Minneapolis, Minnesota, USA \\ *Corresponding author: \\ Tel.: (+1) 860-450-0428 \\ Email: chandi.witharana@stonybrook.edu
}




\begin{abstract}
Remote sensing is a rapidly developing tool for mapping the abundance and distribution of Antarctic wildlife. While both panchromatic and multispectral imagery have been used in this context, image fusion techniques have received little attention. We tasked seven widely-used fusion algorithms: Ehlers fusion, hyperspherical color space fusion, high-pass fusion, principal component analysis (PCA) fusion, University of New Brunswick fusion, and wavelet-PCA fusion to resolution enhance a series of singledate QuickBird-2 and Worldview-2 image scenes comprising penguin guano, seals, and vegetation. Fused images were assessed for spectral and spatial fidelity using a variety of quantitative quality indicators and visual inspection methods. Our visual evaluation elected the high-pass fusion algorithm and the University of New Brunswick fusion algorithm as best for manual wildlife detection while the quantitative assessment suggested the Gram-Schmidt fusion algorithm and the University of New Brunswick fusion algorithm as best for automated classification. The hyperspherical color space fusion algorithm exhibited mediocre results in terms of spectral and spatial fidelities. The PCA fusion algorithm showed spatial superiority at the expense of spectral inconsistencies. The Ehlers fusion algorithm and the wavelet-PCA algorithm showed the weakest performances. As remote sensing becomes a more routine method of surveying Antarctic wildlife, these benchmarks will provide guidance for image fusion and pave the way for more standardized products for specific types of wildlife surveys.
\end{abstract}

Keywords: penguins, seals, guano, data fusion, VHSR imagery 


\section{Introduction}

\subsection{Remote sensing for Antarctic wildlife}

Since its discovery, the Antarctic has been plagued by data scarcity, as geological and biological surveys have been largely confined to easily-accessed coastal regions, or to the areas surrounding permanent Antarctic stations capable of providing logistical support to survey operations. However, the Antarctic is quickly becoming a model system for the use of remote sensing for the physical and biological sciences because the costs of field access are high, the landscape is simple (e.g., rock, snow, ice, water) and free of woody vegetation, and polar orbiting satellites provide extensive regular coverage of the region. Increased access to sub-meter commercial satellite imagery, which rivals aerial imagery in terms of spatial resolution, has sparked a number of developments in geology, glaciology, geography, oceanography, and biology. Very high spatial resolution (VHSR) satellite sensors like IKONOS, QuickBird, GeoEye, Pléiades, Worldview-2, and Worldview-3 provide very high resolution multi-spectral imagery that can capture the detail needed for an array of applications, e.g. individual houses on a city street, individual animals standing on the ground, or individual tree canopies within a forest stand (Ardila et al., 2012; Kurtz et al., 2012; Lynch et al., 2012; Beguet et al., 2014; Huang et al., 2014; Karlson et al., 2014). Due to shorter revisit times of these sensors, it is also possible to acquire near real-time imagery over the area of interest (Kim et al., 2011).

While remote sensing has a long and fruitful history of use mapping environmental layers such as land cover type, which can be used as an indirect measure of biodiversity, the use of remote sensing imagery to directly survey animals (either individual animals, or groups of animals) is, with a few notable exceptions (Schwaller et al., 1983; Schwaller et al., 1989), a much more recent phenomenon (BarberMeyer et al., 2007, Fretwell and Trathan, 2009; LaRue et al., 2011, Lynch et al., 2012). Recent satellitesupported surveys of wildlife in the Antarctic have included penguins (Pygoscelis spp. and Emperor Penguin, Aptenodytes forsteri; Barber-Meyer et al., 2007; Fretwell and Trathan, 2009; Fretwell et al., 2012; Lynch et al., 2012; Schwaller et al., 2013; LaRue et al., 2014; Lynch and LaRue, 2014; Fretwell et 

seal, Mirounga leonina, McMahon et al., 2014), and whales (Abileah, 2002; southern right whale, Eubalaena australis; Fretwell et al., 2014).

VHSR satellite sensors typically record image data in a low resolution multispectral (MS) mode and high resolution panchromatic (PAN) mode. High spatial resolution is needed to accurately describe feature shapes and textural patterns, while high spectral resolution is needed to classify detailed land-use and land-cover types (Wald, 2000; Rachin et al., 2003; Ehlers et al., 2010; Myint et al., 2011; Yuhendra et al., 2012). Fusing PAN and MS images with complementary characteristics can provide a better representation of the observed area (Wald, 2000; Ranchin et al., 2003). In this respect, data fusion serves as an integral step in the processing of remotely sensed imagery for ecological applications. This is particularly true in surveys of wildlife, where classification hinges on both spectral and spatial information.

The increasing availability of VHSR imagery has resulted in the need for more sophisticated image processing techniques (Gamba, 2014; Pohl and van Genderen, 2014; Xu et al., 2014; Zhan, 2014). Over the years, many image fusion methods have been developed and tested (Hallada and Cox, 1983; De Bethune et al., 1998; Zhan, 2002; Ehlers et al., 2010). A fusion algorithm that preserves the spectral properties of the MS data and the spatial properties of the PAN data would be ideal, but there is always a compromise between these two elements (Civco et al., 2009). The choice of fusion algorithm depends on the application domain because the reflectance varies with different environmental features. Data fusion algorithms introduce spectral and spatial distortions to the resulting fused product that depend on the scene content; therefore, a careful selection of fusion method is required. For example, a fusion algorithm designed to address high-frequency edge information in urban landscapes might not produce satisfactory results when applied to a relatively homogenous agricultural or forested landscape (Witharana et al., 2013b). In this respect, it is challenging to transfer the knowledge on the performances of fusion algorithms that have been tested in one application domain to another application domain. There is a plethora of literature on fusion-quality assessments addressing general context (Vijayaraj et al., 2006; 


\subsection{Image fusion and quality assessment}

Pohl and van Genderen (1998) defined image fusion as a tool to combine multisource imagery using advanced image processing techniques that can be performed at three different processing levels (pixel, feature, and decision) depending on the stage at which the fusion takes place. Image fusion can occur in different ways such as inter-sensor, intra-sensor, single-date, and multi-date. Pansharpening, also called resolution merge (Gangkofner et al., 2008) is a pixel-level fusion technique used to increase the spatial resolution of the multispectral image while preserving the spectral information (Vijayaraj et al., 2006). Many studies report the problems and limitations associated with different fusion techniques (Chavez et al., 1991; Wald et al., 1997; Zhang, 2002). The most frequently encountered problem in fusion algorithms is that the fused image exhibits a notable deviation in visual appearance and spectral values from the original MS image (Ling et al., 2007; Kalpoma and Kudoh, 2007). Spectral distortions including spatial artifacts affect both manual and automated classifications because any error in the synthesis of the 
spectral signatures at the highest spatial resolution incurs an error in the decision (Ranchin et al., 2003).

Thus, it is necessary to evaluate the quality of fused images in terms of qualitative and quantitative indices. Qualitative comparison of the fused image and the original MS and PAN images for color preservation and spatial improvements is the most simple but effective way of benchmarking different fusion algorithms (Nikolakopoulos, 2008); on the other hand, visual inspection methods are also subjective and largely depend on the experience of the interpreter (Klonus and Ehlers, 2007; Ehlers et al., 2010).

A number of objective metrics have been proposed to quantify spectral and spatial distortions incurred during the fusion process. Li et al. (2010) document a comprehensive survey on spectral quality indices. The most widely used metrics for evaluating spectral fidelity are the two-dimensional correlation coefficient (CC), root mean squared error (RMSE), relative difference of means, relative variation, deviation index, and band discrepancy. Research by Vijayaraj et al. (2006), Karathanassi et al. (2007), and Yakhdani and Azizi (2010) used peak-signal-to-noise ratio (PSNR) and entropy as spectral quality metrics in addition to common indicators. Wald (2000) proposed the ERGAS metric, which aims to provide a quick but accurate measure of the overall quality of a fused product. Samadzadegan et al. (2012), Witharana et al. (2013b), and Liu et al. (2014) used the spectral angle mapper (SAM) to assess the overall spectral quality of fused images. The universal image quality index (Q-average) is a global metric that models any distortions as a combination of loss of correlation, luminance distortion, and contrast distortion (Wang and Bovik, 2002). Alparone et al. (2004) generalized the Q-average as the Q-4 index, which Alparone et al. (2007) applied to assess fusion quality along with SAM and ERGAS. Alparone et al. (2006) proposed a new index called QNR based on the findings of Wang and Bovik (2002) and Xydeas and Petrovic (2000). The correlation of the gradient information, a combined quantity to evaluate spectral consistency and information content, was developed by Weidner (2010) based on the findings of Wang and Bovik (2002) and Xydeas and Petrovic (2000). Wang et al. (2004) proposed another metric called mean structure similarity index (MSSIM), which is an enhanced version of the Q-average. Ling et al. (2007) and Ehlers et al. (2010) adopted the MSSIM to evaluate the spectral fidelity of fused images. 
Compared to spectral quality indicators, only a few metrics are available to evaluate the spatial fidelity of fused images (Makarau et al., 2012). Ehlers et al. (2010), Gangkofner et al. (2008), Klonus and Ehlers (2007), and Yakhdani and Azizi (2010) used high-pass correlation and edge detection using filters like Canny, Sobel, and Perwitte. In contrast, Civco et al. (2009), Civco and Witharana (2012), and Witharana et al. (2013a) tested a new approach based on Fast Fourier Transform (FFT). Guo et al. (2010) used another metric called average gradient index that describes the changing features of image texture and detailed information to evaluate the spatial quality of fused images.

The remainder of this paper is structured as follows. Section 2 describes the study area, image data, fusion algorithms, and evaluation methods. Section 3 reports the spatial and spectral fidelity of fused products in terms of quantitative indices and visual inspections. Section 4 contains a discussion explaining the results based on the performances of fusion algorithms and proposes a suite of suggested methods for manual and automated ecological feature extraction in EO-based Antarctic monitoring studies. Finally, conclusions are drawn in Section 5.

\section{Materials and methods}

\subsection{Study area and image data}

In this study we used a set of cloud-free QuickBird-2 and Worldview-2 images comprising penguin (Pygoscelis spp.), seal, and shag (Phalacrocorax spp.) colonies previously surveyed on the ground by the Antarctic Site Inventory (ASI; Lynch et al. 2013) (Figure 1). Images were obtained during the austral summer during 2002-2014. Candidate scenes were radiometrically corrected, orthorectified, and spatially registered to WGS 84 datum and Stereographic South Pole projection. The QuickBird-2 sensor has a ground sampling distance (GSD) of $0.61 \mathrm{~cm}$ for the PAN and $2.44 \mathrm{~m}$ for MS bands at nadir with 11-bit radiometric resolution. The Worldview-2 hyperspatial sensor records the PAN and the 8 MS bands with a GSD of $0.46 \mathrm{~m}$ and $1.84 \mathrm{~m}$ at nadir, respectively, with 11 bit radiometric resolution (Table 1). 


\subsection{Methods}

From the candidate image scenes, we extracted 50 non-overlapping subsets (each approximately $2 \mathrm{~km} \times 2 \mathrm{~km}$ ) for the detailed fusion evaluation. The selection of subsets was made based on three target classes: penguin guano, seals, and vegetation, which are those targets most fully developed for satellitebased Antarctic wildlife monitoring. We used seven data fusion algorithms (Table 2) commonly encountered in the literature and incorporated into commercial remote sensing image processing software packages (e.g., ERDAS Imagine 2014, ENVI 5.2, and PCI Geomatica 2014). The underlying principles of the candidate algorithms are briefly described below and we refer the reader to the literature listed in Table 2 for detailed information. All fusion techniques were performed at their default settings to preclude the effect of the parameter optimization process on individual algorithms and to ensure an unbiased quality assessment. Fusion results were assessed using a series of quality metrics along with detailed visual inspection procedures to benchmark the spectral and spatial fidelity of fused products compared to their original MS and PAN images.

$<$ Table $2>$

\section{Ehlers fusion algorithm}

The Ehlers (EH) fusion algorithm is based on an intensity-hue-saturation (IHS) transform coupled with adaptive filtering in the Fourier domain (Klonus and Ehlers, 2007). The main design goal of the EH algorithm is to enhance the spatial resolution of the MS image without adding new grey level information to its spectral components. The EH algorithm entails a twofold workflow: 1) separation of spectral and spatial information and 2) manipulation of spatial information content in a way that allows an adaptive enhancement of the images. The MS image is subjected to an IHS transform for an optimal separation of spectral information. A Fast Fourier Transform (FFT) is then applied to the intensity component and the PAN image, which facilitates an adaptive filter design in the frequency domain. A low-pass and a highpass filter are used to filter intensity spectrums and high-frequency information from PAN, respectively. 
Finally, an inverse FFT is tasked to transform the images to the spatial domain with complementary frequency information from the MS and PAN images and the new intensity component is inversely transformed combining the original hue and saturation components of the MS image (Ehlers et al. 2010).

\section{Gram-Schmidt fusion algorithm}

The Gram-Schmidt (GS) method is a component substitution method (Nikolakopoulos, 2008), which is, in principle, analogous to the principal component analysis fusion technique. The GS technique transforms a set of vectors into a new set of orthogonal and linear independent vectors (Ehlers et al. 2010). First, the low spatial resolution MS spectral bands are averaged to simulate a low resolution PAN band. Next, the GS orthogonalization transformation is performed for the simulated PAN band and the MS band, where the simulated PAN band applied as the first band. The original high frequency PAN band is then swapped with the first GS component. Finally, an inverse GS transform is applied to form a resolution-enhanced MS image (Laben et al. 2000).

\section{Hyperspherical color space fusion algorithm}

The hyperspherical color space (HCS) fusion technique, which taxonomically falls into the 'component substitution' class of fusion methods, is specifically designed for enhancing WorldView-2 8band images, though it is capable of pansharpening any MS data containing 3 or more bands (Padwick et al. 2010; ERDAS, 2014). The main task involved in the HCS methods is the transformation (forward and reverse) of data from native color space to hyperspherical color space. An image with $\mathrm{N}$ input bands yields a single intensity component and N-1 angles on the hypersphere. Thus, within the hyperspherical color space, the single intensity component can be scaled without distorting the color. In the first pass, mean and standard deviation of the square of the PAN image and the intensity component of the MS image are computed. Then a forward transformation is applied to the intensity component from the native color space to the hyperspehrical color space. Pansharpened intensity is obtained by matching the signals from the square of PAN and the intensity component. Next the matched intensity is swapped with the 
original intensity component of the MS image and a reverse transformation is performed from the hyperspherical color space to the native color space.

\section{High-pass filter fusion algorithm}

The High-pass filter (HPF) method is a spatial domain fusion technique. The image synthesis process involves a convolution using a high-pass filter on the high resolution data and then combining the filtered frequencies with the lower resolution MS data (Gangkofner et al. 2008). The algorithm has three key steps. First, the HP filtering is applied to the PAN image to extract the structural detail. Then, the HPfiltered image is combined with each of the MS bands using a standard deviation-based injection model. Finally, a linear histogram matching is tasked to adapt the standard deviation and mean of the synthesized image bands to those of the corresponding original MS Image.

\section{Principal component analysis fusion algorithm}

Principal Component Analysis (PCA) is a statistical method that transforms a multivariate dataset of correlated variables into a dataset of uncorrelated linear combinations of the original variables. In terms of its relationship to other data fusion methods, PCA methods fall into the component substitution class. First, the MS image is converted into a new set of uncorrelated components and the first PC is extracted assuming that it contains only the overall scene luminance (Karaksanthi et al. 2007). Next the PAN image is histogram matched to the first PC and then substituted with the selected component. Finally, an inverse PC transform projects the fused dataset back to the original MS feature space (Ehlers et al. 2010).

\section{University of New Brunswick fusion algorithm}

The University of New Brunswick (UNB) technique is a statistic based fusion method (Zhang 2002, Zhang, 2004). UNB fusion adapts a least squares approach to approximate the grey value relationship between the original MS, PAN, and fused images to achieve an optimal color representation that aims to address two fundamental drawbacks inherent to image fusion algorithms: 1) color distortion and 2) 


\section{Wavelet transform fusion algorithm}

The Wavelet transform (WV) technique, a spatial domain fusion method, extracts the high-frequency detail coefficients from the high spatial resolution PAN image and combines it with the spectral information obtained from the MS image using a combination model (Vijayaraj et al. 2006; Ehlers et al. 2010). First, three new PAN images are produced based on the histogram of the MS image. Next, each synthesized PAN image is decomposed into a low-resolution approximation image and wavelet coefficients (detail images) comprising spatial information. Next the decomposed low-resolution PAN images are then substituted with the original MS image bands. Finally, a reverse wavelet transform is applied to each of the three sets containing the local spatial details and one of the MS bands to inject highresolution spatial details from the PAN image to the low-resolution MS image.

\subsubsection{Quantitative evaluation}

Objective quality assessments can be either direct or indirect. Whereas the former gauges the quality of the fused imagery itself, the latter assesses the quality of products extracted from the fused imagery. We chose the direct approach, which entailed eleven spectral and four spatial metrics, each calculated independently for each subset and separately for each band (except for ERGAS and SAM). Use of eleven spectral and three spatial metrics, totaling 14 objective quality indicators in our evaluation procedure, might be questionable because these metrics might be redundant and correlated (Pradhan et al. 2006; Gangkofner et al. 2008; Li et al., 2010; Jawak and Luis 2013; Witharana et al., 2013). Previous 
researchers used different combinations of quality indicators to rank fusion algorithms (Vijayaraj et al., 2006; Karathanassi et al., 2007; Ehlers et al., 2010; Kim et al., 2011; Witharana et al., 2013), however these studies found no consistent suite of quality metrics that worked for all ground targets. We employ a full complement of objective quality indicators to reexamine their stability and redundancy, and to investigate the dependency of the ranking of fusion algorithms on quality metrics. These metrics' mathematical and statistical bases are summarized in Table 3. The fusion-evaluation workflow tasked in this study is depicted in Figure 2.

\title{
2.1.1 Visual inspection
}

Four remote sensing experts, two interpreters with domain-specific (Antarctic wildlife monitoring; HJL and MAL) manual image-interpretation skills and another two with general land use/cover mapping expertise, were tasked to inspect the color preservation and spatial improvement of fused images with respect to their parent MS and PAN images. For each sensor, we chose eight test sites for each target class, totaling 22 zoomed-in views. We selected false-color composites (bands green, red, and near infrared [NIR] for QuickBird images and bands green, red, and near infra red1 [NIR1] for Worldview-2 images) for visual inspections because this band combination is widely used for many remote sensing applications (Ehlers et al., 2010; Witharana et al., 2013; Witharana et al., 2014). False-color composites along with their original images were inspected by all the experts to identify spectral distortions (e.g., brightness reversions, saturation, a complete change of spectral characteristics, unnatural/artificial colors) and spatial improvement in general context. When benchmarking pansharpening quality, the domainspecific experts specifically focused on spectral and spatial clarity (e.g. counting of seals at the individual level and delineation of penguin guano based on color and texture) of wildlife elements in fused images.

\author{
$<$ Table $3>$ \\ $<$ Figure $2>$
}




\section{Results}

3.1 Visual analysis

Figures 3-5 and Figures 6-8 depict six representative test scenes acquired by QuickBird and Worldview-2 sensors, respectively. The experts were asked to report the best two and the worst two fusion algorithms for each target class. Rank ordering of all algorithms would have been ideal; however, in reality, ranking of seven fusion algorithms (i.e., best to worst) based on their performances is a difficult task even for an expert photointerpreter because, while the human brain easily discriminates among extreme variations (i.e., the best and worst fused images), it fails to distinguish subtle variations among images with similar spectral and spatial properties.

Table 4 reports the subjective rating of pansharpening quality from the viewpoints of domain-specific wildlife detection (Analysts 1 and 2) and general land cover mapping (Analysts 3 and 4). With only one exception (Analyst 2's rating on guano), all experts elected the HPF and UNB fusion algorithms best for all three target classes and the EHL and WVL algorithms as the worst. Inspecting representative scenes of QuickBird (Figure 3) and Worldview-2 (Figure 6) scenes containing penguin guano, we see that the HPF fusion algorithm has clearly improved the spatial resolution of the MS image while improving edge information and injecting texture features, which are innate to penguin colonies, from the PAN image. The resulting high contrast images show some spectral degradation, however, the spatial improvement compensates for losses in color fidelity. The HPF product is more visually efficient than its main contender (the UNB fusion algorithm) since it expediently signals rich visual cues to the observer to spot the presence of guano and delineate its spatial extent. The EHL and WVL algorithms have preserved the spectral character of the original MS images at the expense of spatial improvement. No edge improvement can be seen in EHL and WVL products. The GSM and PCA fusion algorithms exhibited mediocre visual quality. In the case of seal haul outs, the HPF fusion algorithm provides valuable visual cues to detect individual seals on the beach (Figure 7); however, it has introduced spatial artifacts to the fused scene comprising seals on pack ice (Figure 4). The HPF algorithm has over-emphasized the surface 
undulations, which ultimately lead to dark pseudo blobs that are visually analogous to seals and thus problematic for seal census. Of the seven algorithms, the WVL fusion method shows the worst spatial improvement with zero injection of high frequency information from the PAN image to the MS image, which precludes the individual detection of seals. Visual analysis of QuickBird (Figure 5) and Worldview-2 (Figure 8) scenes comprising vegetation reveal that the EHL fusion algorithm has produced distinct spatial artifacts while the PCA fusion algorithm has introduced serious color distortions (color reversion). Similar to previous cases, the HPF fusion algorithm exhibits significant edge enhancement with some spectral degradation. The GSM and UNB algorithms show mediocre visual quality while mitigating both spectral and spatial distortions. Figure 9 shows a set of zoomed-in Canny-edge filtered images of the original WV-2 images and fused products. The WVL, EHL, and HCS fusion methods show weak performances in injecting high frequency information from the PAN image to the MS image. Noticeably, the WVL's filtered image shows fuzzy and poorly improved edges when compared to its parent images. In general, the edge information embedded to the filtered images of the GSM, UNB, and HPF methods exhibit a promising agreement with the structural information encoded to their parent images.

$<$ Table 4 >

$<$ Figures 3, 5, 6, 7, 8, and 9>

\subsection{Quantitative assessment}

We corroborated visual assessment with eleven spectral metrics and four spatial metrics. Figures 1012 and Figures 13-15 depict the summary plots constructed based on the objective metrics for the fused images of QuickBird and Worldview-2, respectively.

When analyzing the quality plots of the QuickBird scenes with penguin guano (Figure 10), the basic spectral metric - correlation coefficient (CC) - reports the best scores for the WVL, EHL, and HCS fusion algorithms indicating discernible band-wise agreement between fused and original MS images. While the GSM, PCA, and UNB methods reported comparable results, the HPF method emerged as the worst with respect to the CC. The plots of root-mean-squared-error (RMSE), spectral discrepancy (SD), and 
divination index (DI) reported high scores for EHL, HPF, and PCA fusion algorithms indicating degraded spectral fidelity. The plots of relative distance to mean (RDM) and the relative distance to standard deviation (RDS) clearly manifest the underlying histogram shift in the EHL and PCA fused products. With respect to the peak signal-to-noise ratio (PSNR) and entropy difference, the WVL and the HPF methods had the least and greatest radiometric distortions, respectively. The EHL fusion algorithm showed low structural similarity measure (SSIM) scores with high variability. The low SSIM indicates the loss of illumination, radiometric alteration, and contrast distortion between the fused and the original MS images. The WVL and the HCS fusion methods yielded the best results and were comparable to each other. Also, the GSM and the UNB methods yielded superior SSIM scores compared to EHL, HPF, and PCA fusion methods. According to the two global measures (ERGAS and SAM), the EHL and the PCA fusion methods had the worst spectral quality preservation character, while GSM, HCS, and UNB methods yielded superior results and were comparable each other. From the viewpoint of spatial fidelity, all four spatial indices have disqualified the WVL fusion algorithm. The best results for the Canny edge correspondence (Canny edge) were found for the PCA, UNB, and GSM fusion methods. A similar trend was observed for the Sobel edge correlation (Sobel-CC), the high-pass correlation coefficient (HP-CC), and the PAN correlation coefficient (PAN-CC). With respect to the Canny edge, the HCS method outperformed the HPF method; however, the opposite was observed for Sobel-CC. The HPF method clearly outperformed the WVL method with respect to two edge detectors (Canny and Sobel); however, HP-CC and PAN-CC metrics indicate the inferior spatial edge enhancement of the HPF method compared to the WVL fusion algorithm.

Figure 13 entails the quality plots of the Worldview-2 scenes with penguin guano. In general, spectral plots are comparable with those of QuickBird scenes (Figure 9). The EHL fusion, the HCS fusion, and WVL fusion methods showed the highest band-wise correlation with the original MS images while the HPF showed the worst CC results. Despite the high CC values, the EHL method yielded the worst results for per-pixel deviation (RMSE), band-wise deviation (SD, DI), histogram shift (RDM, RDS), radiometric fidelity (PSNR, entropy), structural distortion (SSIM), and global indices (ERGAS, SAM). In most cases 
the PCA fusion algorithm yielded results comparable to the EHL method. The GSM, HCS, and UNB methods reported the highest spectral fidelity with respect to most of the spectral indices. Based on all the spatial metrics, the GSM, PCA, and UNB fusion algorithms surpass the others. Except the PAN-CC, the other three spatial metrics manifest the WVL method's deficient spatial improvement character.

Quantitative quality metric plots for the fused QuickBird and Worldview-2 image scenes with seals are shown in Figures 11 and 14, respectively. When analyzing the spectral quality plots of pansharped QuickBird products, the HPF fusion algorithm exhibited noticeably low CC compared to its contenders. The EHL fusion and the PCA fusion methods seemed to be the worst algorithms with respect to spectral distortions measured by RSME, SD, and DI. The histogram-based metrics (RDM and RDS) also reported high values for the EHL and PCA algorithms. Based on the PSNR, the PCA fusion method yielded the worst results while the HCS, GSM, and WVL fusion methods showed a high degree of radiometric fidelity. In terms of retaining image structure, the WVL method reported the best results while HPF method exhibited the worst results for the SSIM metric. Generally, the GSM, HCS, and UNB methods yielded high values for SSIM, proving their ability to conserve the image structure during synthesis. From the view point of global spectral metrics, the PCA and the HPF methods yielded the worst results for the ERGAS and SAM metrics, respectively. Despite the weak spectral performances, the PCA fusion method reported the best results for all spatial quality indicators. Across all dimensions, the WVL fusion method produced the weakest spatial improvement. In terms of Canny edge correspondence and Sobel edge correlation, the HPF fusion method showed performances comparable to GSM and UNB fusion methods. However, the HPF method yielded inferior results with respect to HP-CC and PAN-CC.

The quality plots based on Worldview-2 scenes (Figure 14) summarize the image synthesis capabilities of candidate fusion methods. With respect to the CC, the WVL, the HCS, and the EHL fusion methods reported the highest spectral congruency between fused and original MS images. However, the EHL method yielded the worst performances (along with PCA fusion method) for RMSE, SD, RDM, and for two global metrics. The HCS algorithm reported stable performances for most of the spectral metrics. It showed low pixel- and band-wise deviation, a high degree of radiometric fidelity, structural 
congruency, and minimal distortion to the shape of the histograms. In many instances, the HPF algorithm emerged as the third worst performing algorithm. From a spatial fidelity perspective, the WVL fusion algorithm exhibited the worst edge enhancement. The two edge-based metrics and the HP-CC have clearly indicated the WVL method's inability to inject spatial structure during synthesis. The GSM, PCA, and UNB methods seemed to be the best performing algorithms. Despite the marked spectral performances, the HCS algorithm showed mediocre results (comparable to the HPF fusion method) for the spatial metrics.

Objective results for the vegetation dominant QuickBird and Worldview-2 image scenes are shown in Figure 12 and 15, respectively. For both sensors, the EH- and PCA-pansharpened images reported the least spectral congruency compared to their contenders. Their radiometric and structural distortions are also apparent when analyzing boxplots of SSIM and PSNR (Figure 12). For the most of the spectral indices derived from QuickBird scenes, the HCS algorithm reported highly satisfactory results. Generally, the GSM and UNB fusion methods have equally conserved the spectral properties during the fusion process. In terms of spatial fidelity, the WVL and PCA algorithms rank as the worst while the GSM, HCS, HPF, and UNB methods produced satisfactory results for the two edge detectors. In the case of Worldview-2 images, the GSM, HCS, UNB, and WVL methods showed equal performances for the most of the per-pixel and band-wise deviation spectral indices. In the case of ERGAS, the HPF fusion exhibited the best results while the EHL and PCA methods reported the worst. Similar to previous cases, the WVL algorithm further proved its systematic failure in injecting high frequency information to the MS image. The EHL and HPF methods showed a relatively weak Canny edge correspondence. Despite the degraded spectral fidelity, the PCA fusion method showed promising results (comparable with GSM and UNB) for the spatial metrics.

$<$ Figures 9,10,11,12,13 and $14>$

\section{Discussion}


Ideally, the fusion process should synthesize the MS image that would have been observed if the multispectral sensor had the spatial resolution of the panchromatic sensor. Data fusion algorithms deviate from this ideal and introduce a variety of spectral and spatial distortions to the modeled image during synthesis. Fusion artifacts may affect subsequent image processing steps like image segmentation, manual image analysis, semi-/fully-automated classification, and other data mining methods. Scene dependency of fusion algorithms impedes the transferability of the knowledge on their performances across application domains (Witharana et al., 2013b). Thus, a comprehensive quantitative evaluation in conjunction with domain expert-led visual gauging of fusion algorithm is necessary to identify those techniques that are optimal with respect to the practical implementation.

Here we report the first detailed study on the performances of data fusion algorithms in support of VHSR-based Antarctic wildlife mapping. We tasked seven widely-used fusion algorithms to pansharpen a series of single-date image scenes acquired by QuickBird-2 and Worldview-2 sensors. We chose penguin guano, seals, and vegetation as target features; of those, the first two have been used in multi-scale EOdriven mapping efforts (Lynch and LaRue, 2014; LaRue, 2014). These early efforts have used either expert visual analysis or expert-derived training datasets because automated classification and segmentation of Antarctic scenes is in its infancy.

From the viewpoints of both subjective and objective evaluations, no single algorithm was able to produce superior results by simultaneously preserving spectral and spatial properties of the original MS and PAN images. Generally speaking, for all target classes, four experts with two different foci (targetspecific inspection and general inspection) rated the HPF and UNB methods as the best fusion algorithms and the EHL and WVL methods as the worst two fusion algorithms. Our quantitative assessment supports the visual rating of the EHL and WVL fusion algorithms as being the worst.

In the objective quality budget, across sensors and target features, the WVL method exhibited superior results at the expense of spatial fidelity. On the other hand, despite the degraded spectral congruency, the EHL and PCA fusion methods yielded better results for spatial metrics. These observations emphasize the necessity of a combined approach (i.e., spectral and spatial fidelity) for 
interpreting fusion results because the best color preservation of an algorithm can be observed even if no pansharpening is performed; on the other hand, a fusion algorithm can achieve the best spatial improvement while producing results with the worst color preservation. The EHL algorithm, which was demonstrated by several studies to be successful (Klonus and Ehlers, 2007; Ling et al., 2007; Ehlers et al., 2010; Ehlers and Klonus, 2014) and was designed specifically for preserving the color information, exhibited poor performances in our study. The overall performance of the PCA fusion algorithm was not consistent: in some instances, it incurred serious color reversions (Figure 5), findings that are in agreement with Witharana et al. (2013b). Despite the spectral superiority, the WVL-sharpened products' spatial fidelity is seriously distorted due to the systematic noise resulting from the shift variance property of the wavelet transform and the filtering procedure. In most cases, both spectral and spatially, the HPF fusion algorithm has been able to surpass the EHL, WVL, and PCA fusion methods. The GSM and UNB fusion methods were able to produce the best quality results by mitigating spatial and spectral artifacts. The validity of the $\mathrm{CC}$ is questionable as it elects the EHL as one of the best-performing algorithm, while all the other indictors disqualify the EHL's spectral fidelity. This reflects the ambiguous nature of quality measures (Makarau et al., 2012) and the necessity of taking a multidimensional assessment.

Padwick et al. (2010) proposed the HCS fusion algorithm with the key design goal of effectively handling the increased spectral information content produced by the Worldview-2 hyperspatial sensor during image synthesis. The HCS-pansharpened images exhibited a marked spectral, radiometric, and structural consistency; however, the spatial consistency was inferior to the GSM and UNB algorithms. A number of studies, such as Zhang (2002), Karathanassi et al. (2007), Yuhendra (2012), Jawak and Luis (2013), and Witharana et al. (2013b) report the success of the GSM and UNB fusion methods. The UNB method serves as the proprietary fusion algorithm for the DigitalGlobe ${ }^{\circledR}$ image products (Zhang 2014). The Polar Geospatial Center (PGC, www.pgc.umn.edu) at the University of Minnesota, which is the key organization engaged in VHSR imagery support for Arctic and Antarctic research, adapts the UNB method as the core pansharpening technique. By design, this algorithm aims to address color distortions 


\section{Conclusion}

Very high spatial resolution satellite remote sensing is being developed as an efficient alternative for traditional, intrusive, time-intensive, and expensive ground and aerial wildlife surveys in Antarctica 
(Fretwell et al., 2012; LaRue et al., 2014; Lynch and LaRue, 2014). Data fusion serves as integral component of remote sensing mapping workflows and is systematically explored in this study. Our visual evaluation determined that the High-pass fusion algorithm and the University of New Brunswick fusion algorithms are optimal for manual feature extraction while the quantitative assessment suggested the Gram-Schmidt fusion algorithm and the University of New Brunswick fusion algorithm for automated classification. The PCA fusion algorithm showed spectral superiority at the expense of spectral inconsistencies. The Ehlers fusion algorithm and the Wavelet-PCA algorithm showed the weakest performances. Further research will be required to 1) explore the fine-tuning capability of the High-pass fusion algorithm, 2) employ an indirect quality assessment workflow to benchmark fusion algorithms, and 3) investigate the synergies of data fusion and the geographic-object based image analysis in the wildlife census applications. 


\section{Acknowledgments}

C.W. and H.L. would like to acknowledge funding provided by the National Science Foundation's Office of Polar Programs (OPP) and Geography and Spatial Sciences (GSS) divisions (Award No.

1255058). We would also like to thank the University of Minnesota's Polar Geospatial Center for imagery processing support. 


\section{List of Figures}

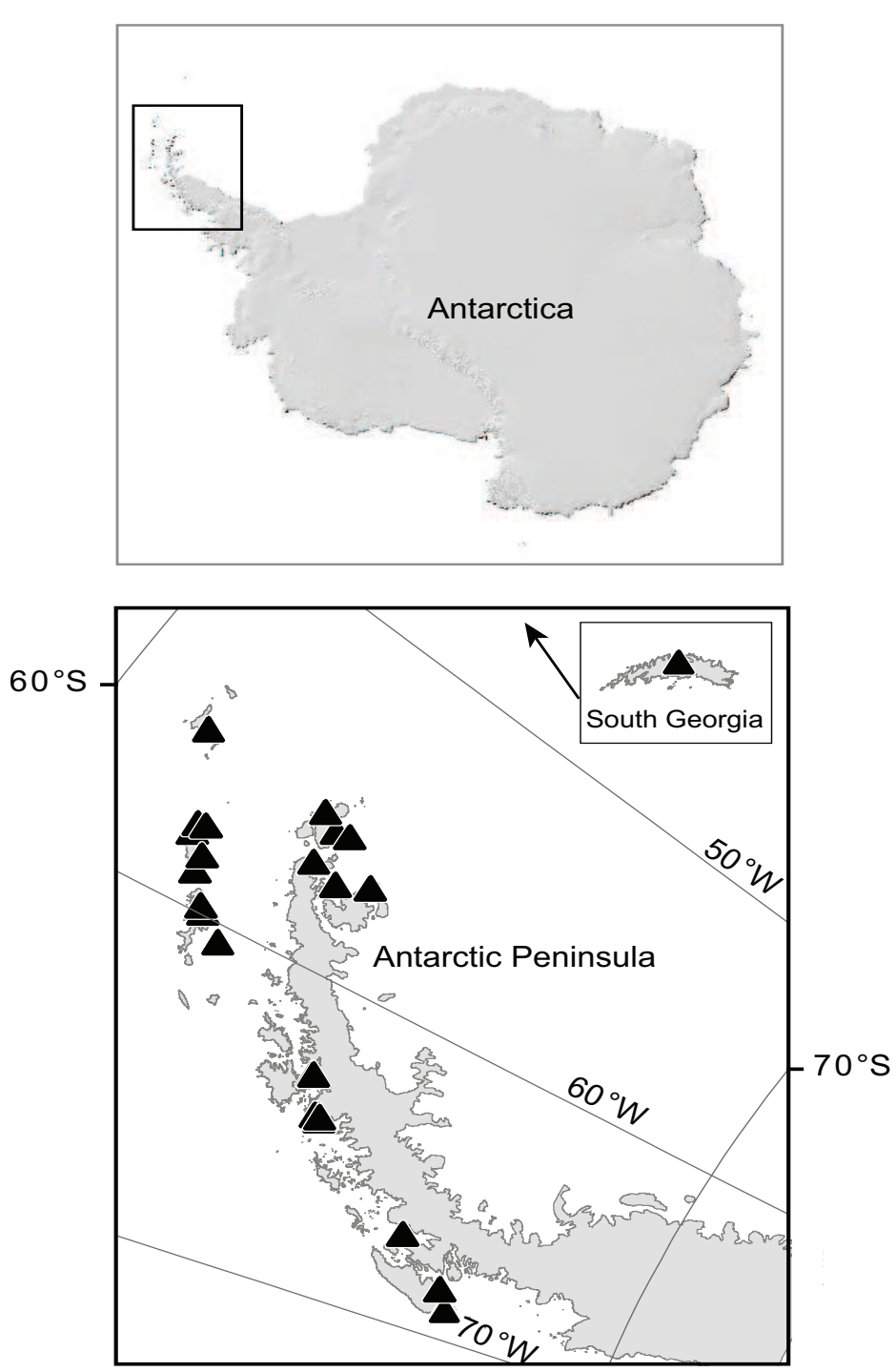

Figure 1. Maps of study area on the Antarctic Peninsula and South Georgia. Black triangles indicate the location of the candidate QuickBird-2 and Worldview2 scenes. 


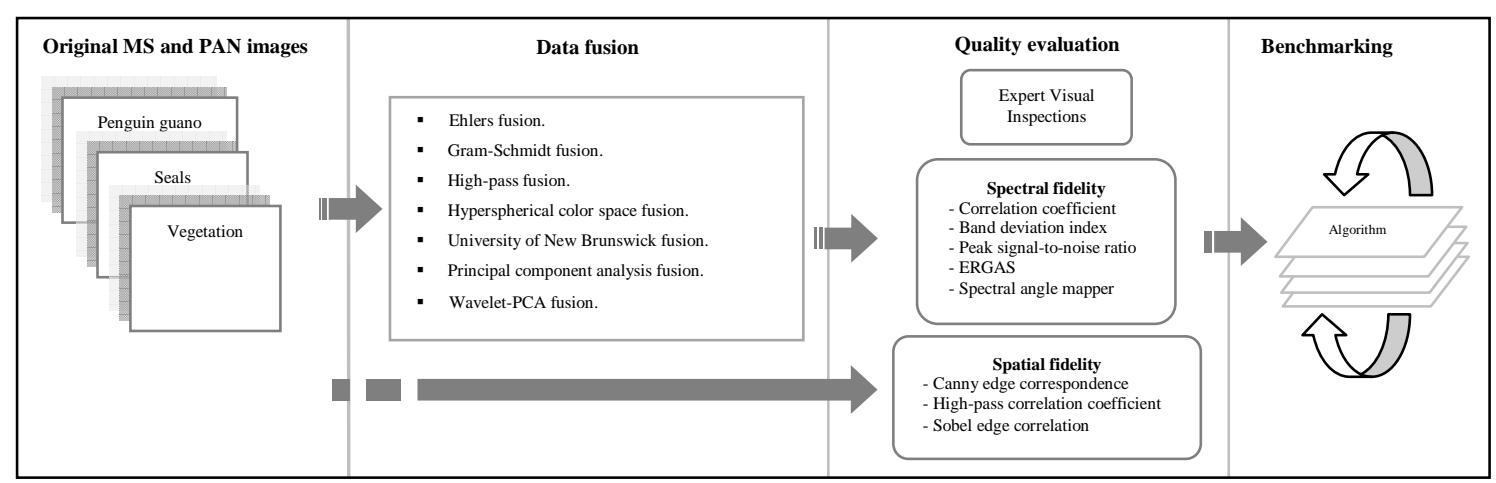

Figure 2. A schematic of the fusion-evaluation workflow 


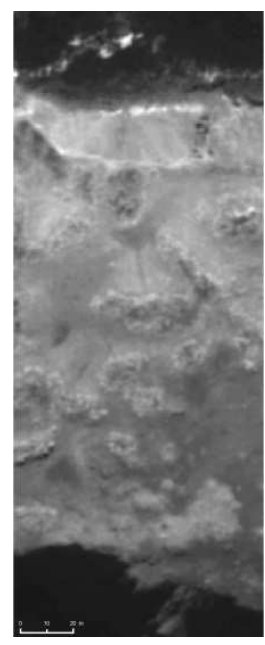

Original PAN image

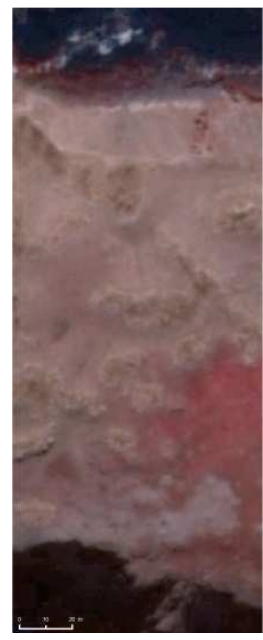

GSM fusion

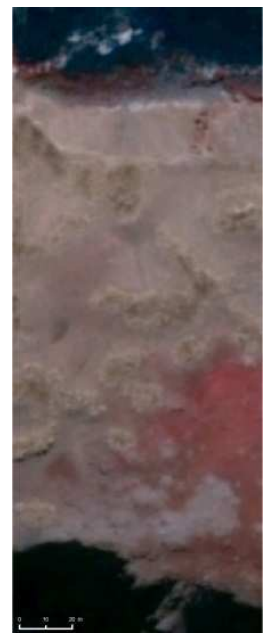

PCA fusion

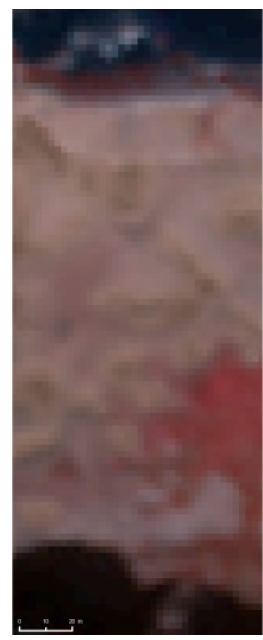

Original MS image

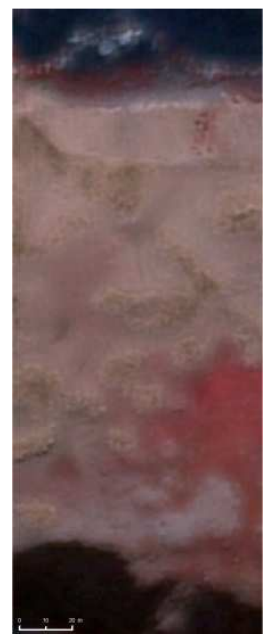

HCS fusion

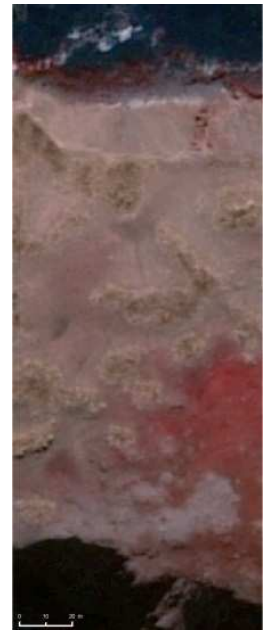

UNB fusion

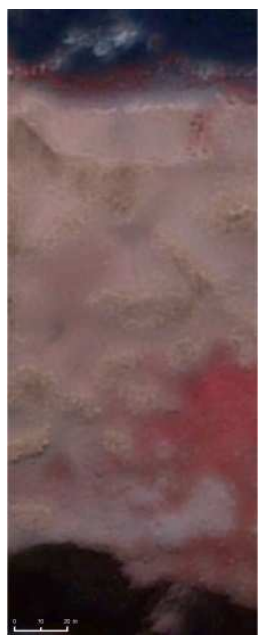

EHL fusion

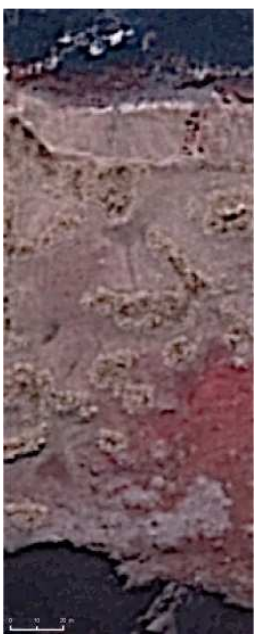

HPF fusion

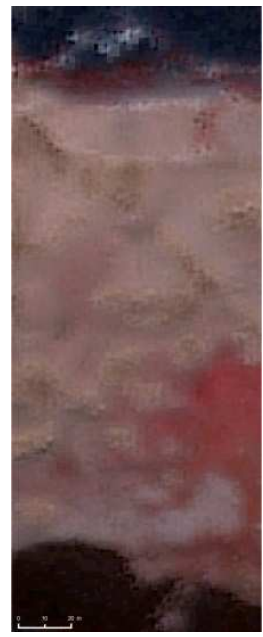

WVL-PCA fusion

Figure 3. A visually inspected site (acquired by QuickBird-2 sensor) for the panshapening quality of fused products comprising penguin guano. Original MS image and fused images are shown as false color (green, red, NIR2) composites. Imagery Copyright DigitalGlobe, Inc. 

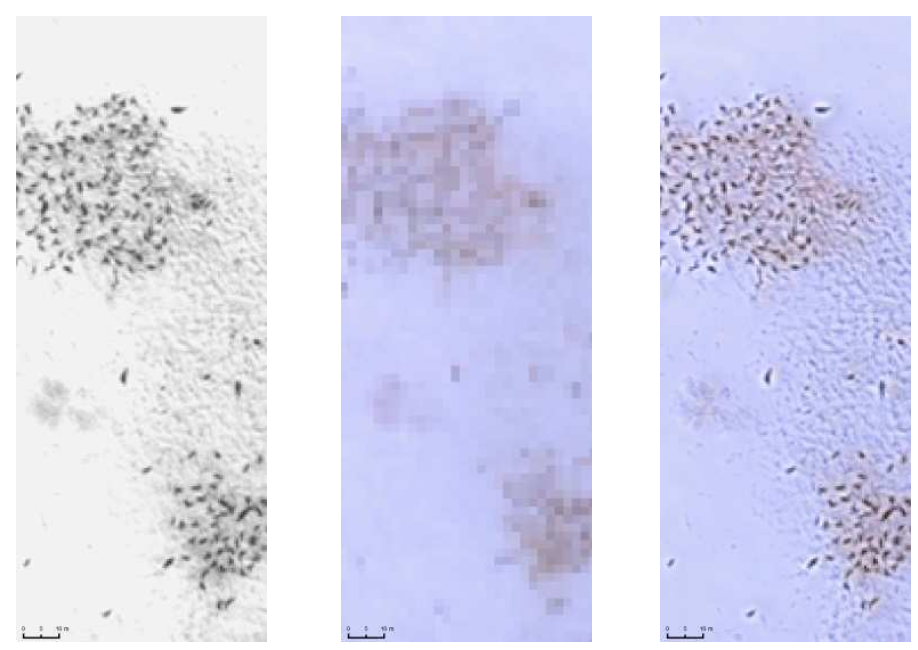

Original PAN image

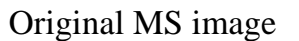

EHL fusion
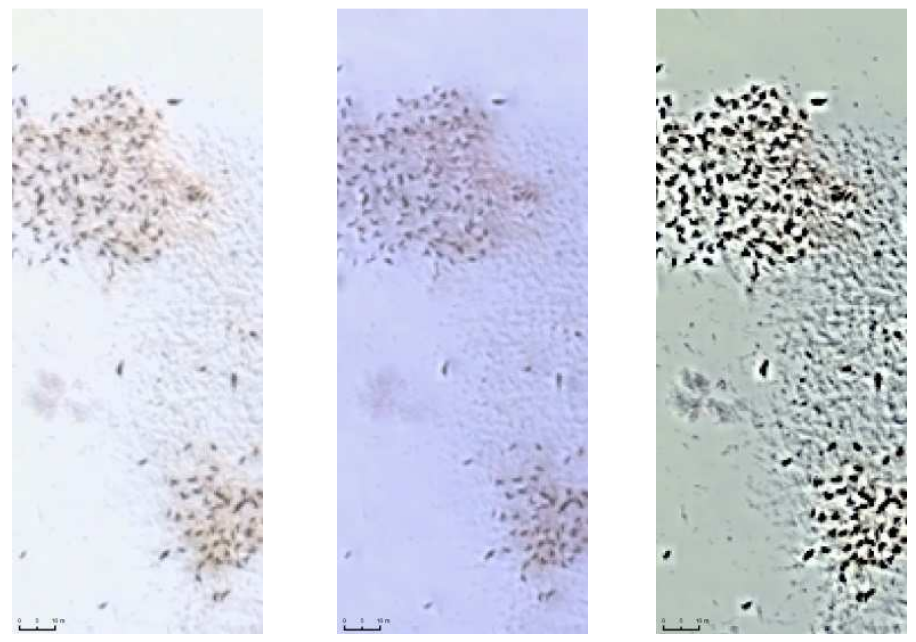

GSM fusion

\section{HCS fusion}

HPF fusion
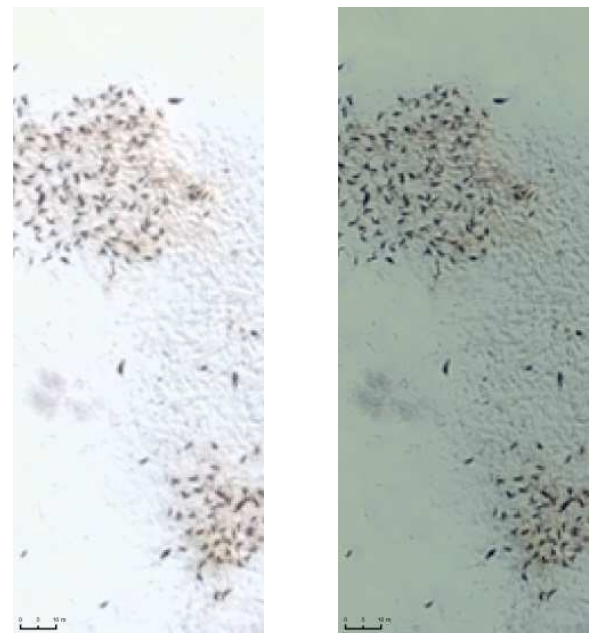

PCA fusion

UNB fusion

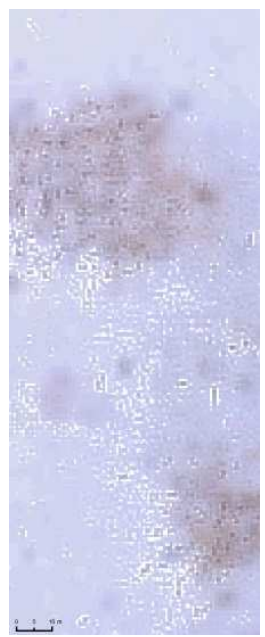

WVL-PCA fusion

Figure 4. A visually inspected site (acquired by QuickBird-2 sensor) for the panshapening quality of fused products comprising seals. Original MS image and fused images are shown as false color (green, red, NIR2) composites. Imagery Copyright DigitalGlobe, Inc. 

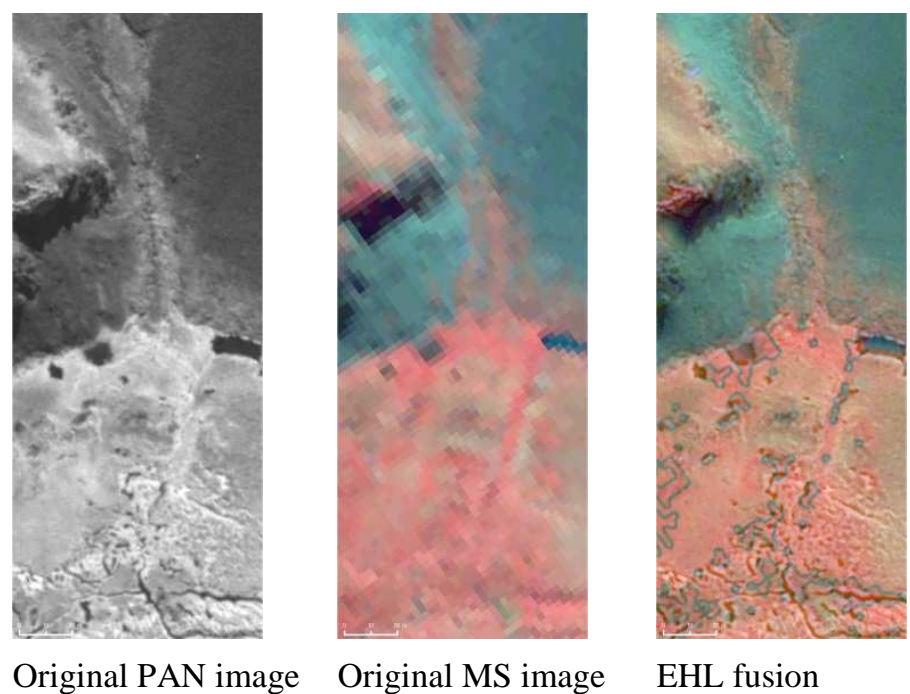

\section{EHL fusion}
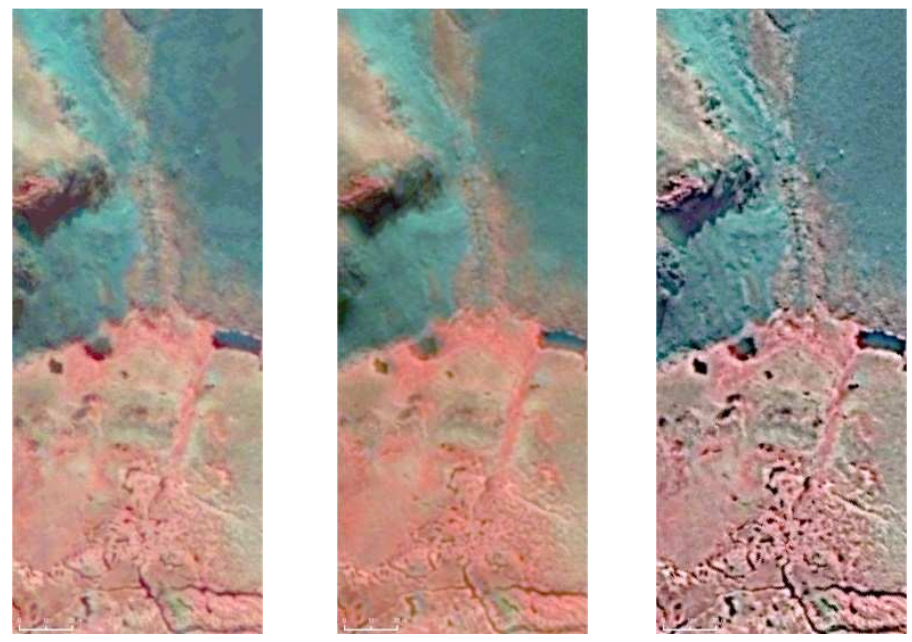

\section{GSM fusion}

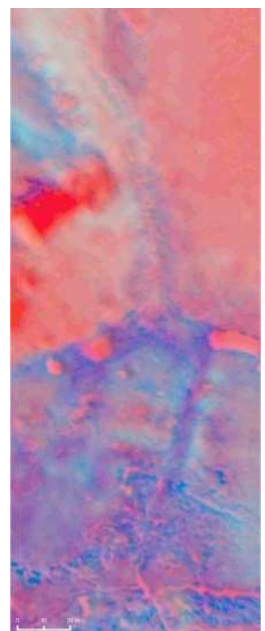

\section{HCS fusion}

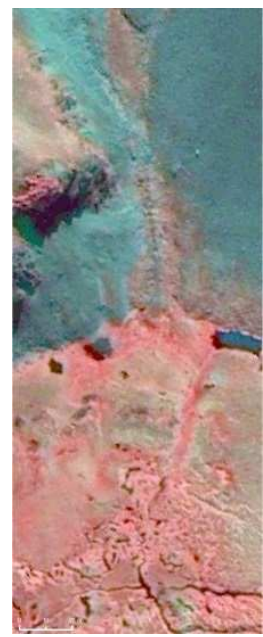

HPF fusion

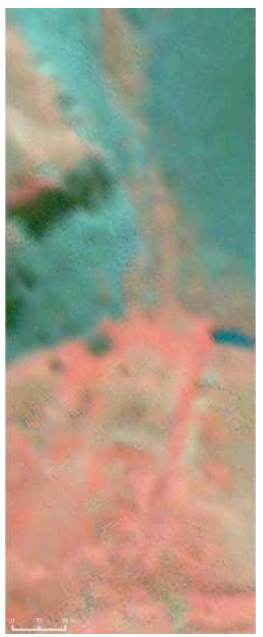

PCA fusion

UNB fusion

WVL-PCA fusion

Figure 5. A visually inspected site (acquired by QuickBird-2 sensor) for the panshapening quality of fused products comprising vegetation. Original MS image and fused images are shown as false color (green, red, NIR2) composites. Imagery Copyright DigitalGlobe, Inc. 


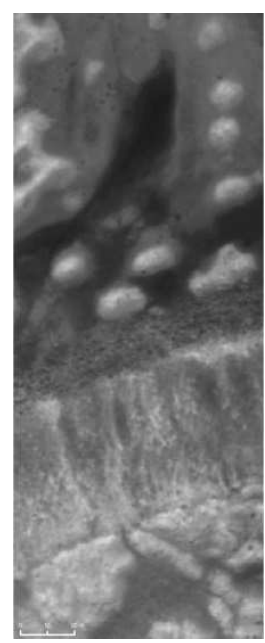

Original PAN image

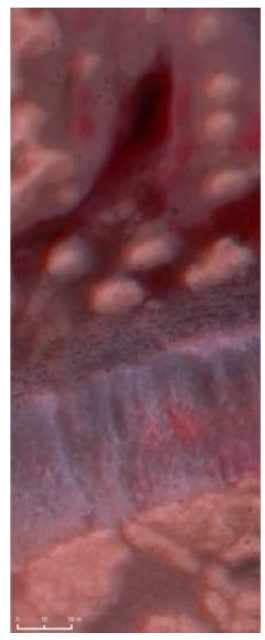

GSM fusion

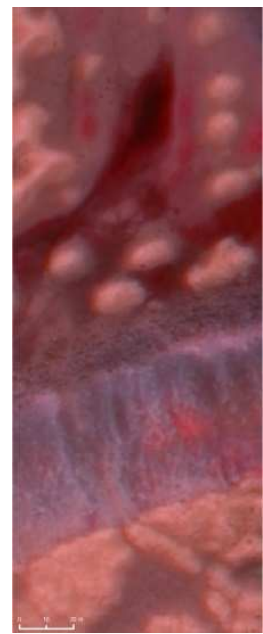

PCA fusion

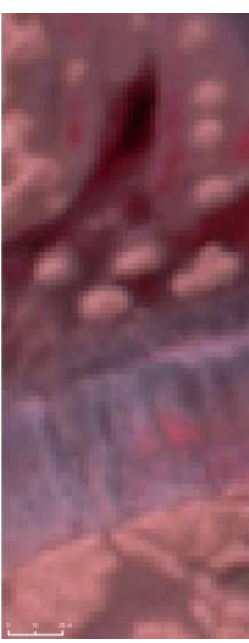

Original MS image

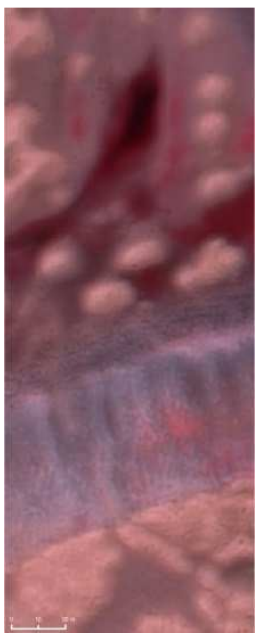

HCS fusion

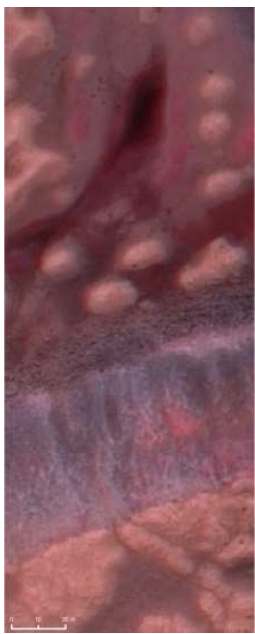

UNB fusion

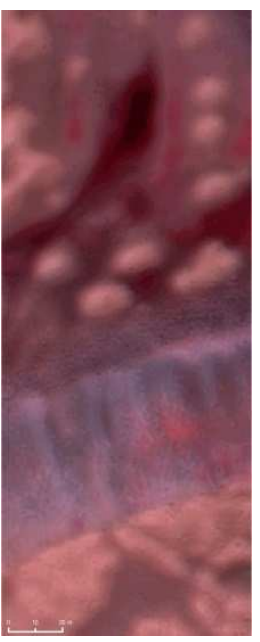

EHL fusion

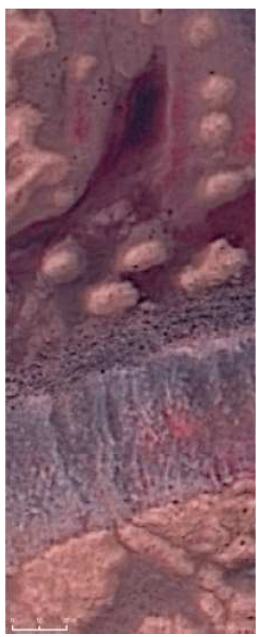

HPF fusion

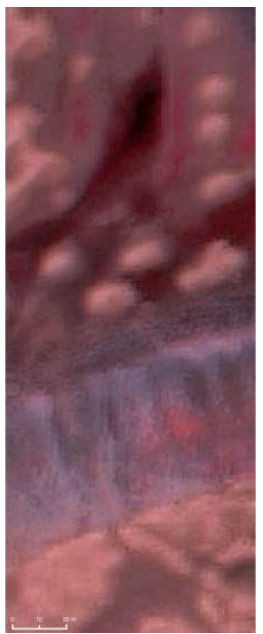

WVL-PCA fusion

Figure 6. A visually inspected site (acquired by Worldview-2 sensor) for the panshapening quality of fused products comprising penguin guano. Original MS image and fused images are shown as false color (green, red, NIR2) composites. Imagery Copyright DigitalGlobe, Inc. 


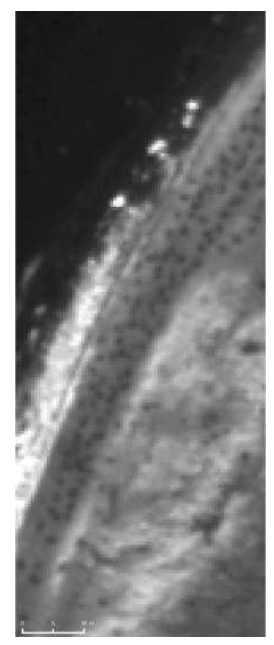

Original PAN image

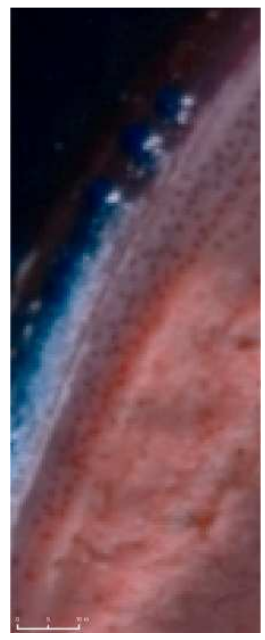

GSM fusion

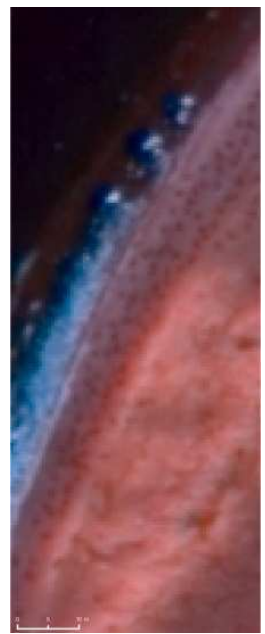

PCA fusion

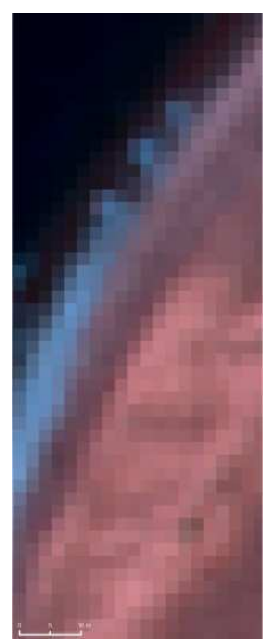

Original MS image

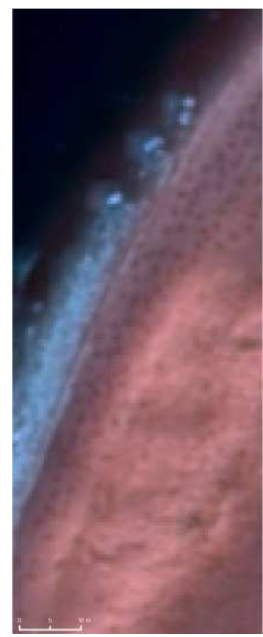

HCS fusion

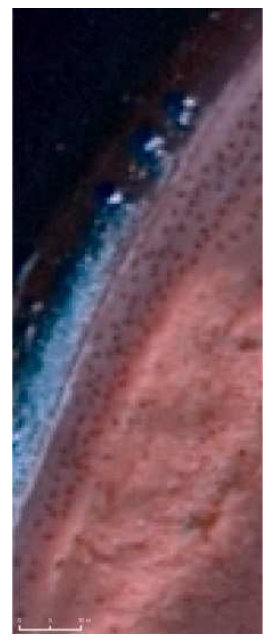

UNB fusion

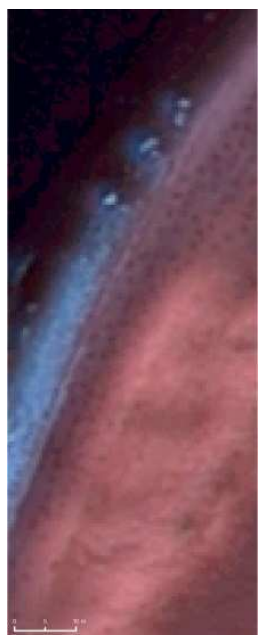

EHL fusion

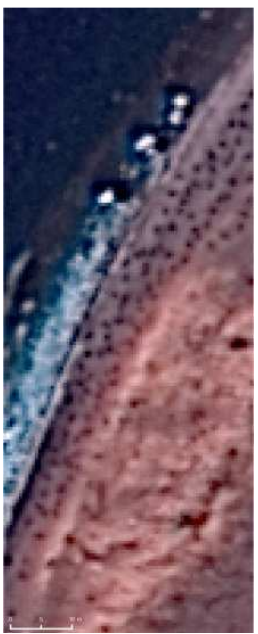

HPF fusion

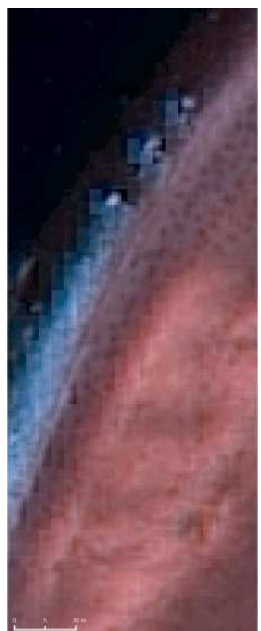

WVL-PCA fusion

Figure 7. A visually inspected site (acquired by Worldview-2 sensor) for the panshapening quality of fused products comprising seals. Original MS image and fused images are shown as false color (green, red, NIR2) composites. Imagery Copyright DigitalGlobe, Inc. 

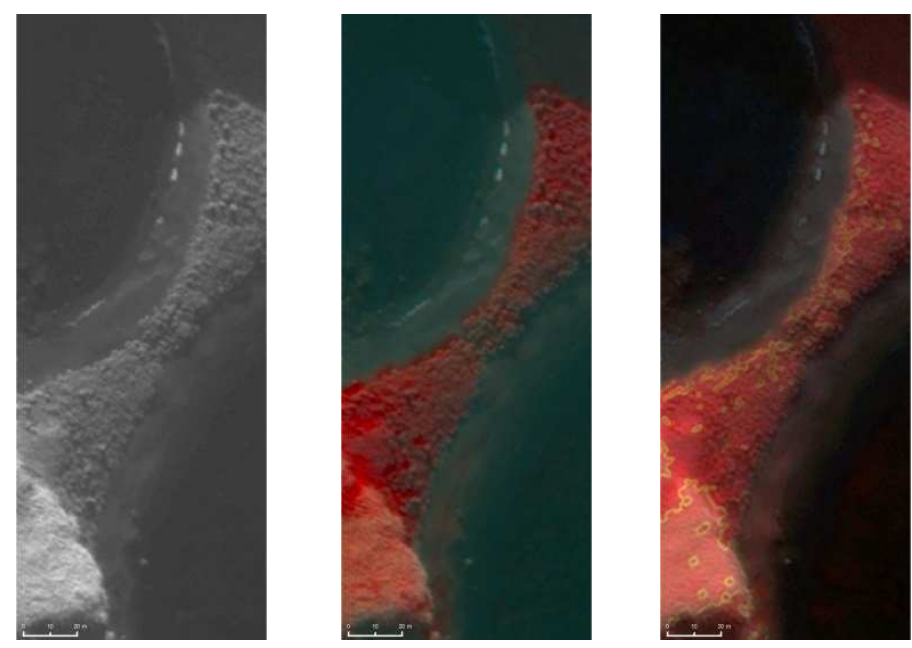

Original PAN image

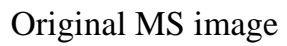

EHL fusion
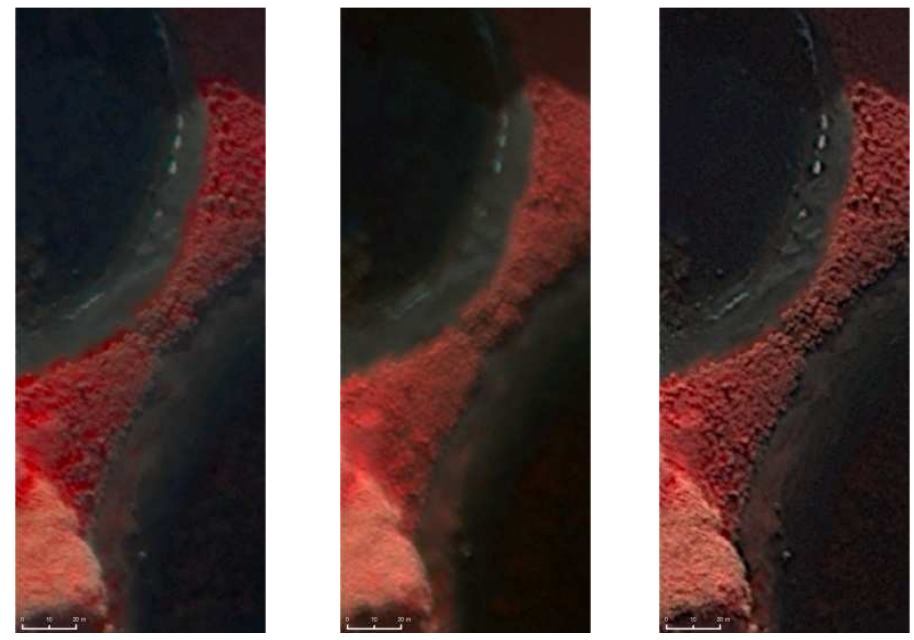

GSM fusion

\section{HCS fusion}

HPF fusion
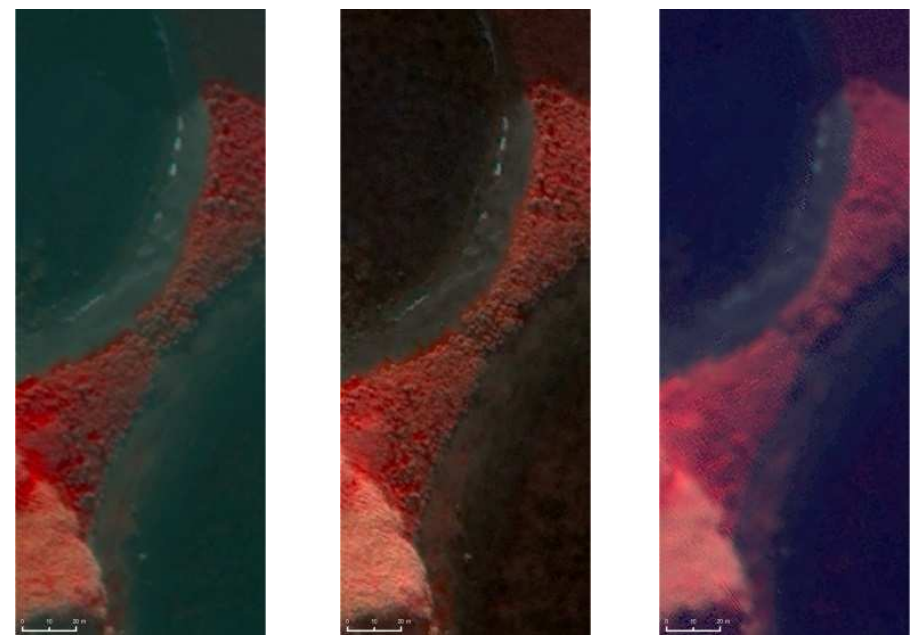

PCA fusion

UNB fusion

WVL-PCA fusion

Figure 8. A visually inspected site (acquired by Worldview-2 sensor) for the panshapening quality of fused products comprising vegetation. Original MS image and fused images are shown as false color (green, red, NIR2) composites. Imagery Copyright DigitalGlobe, Inc. 

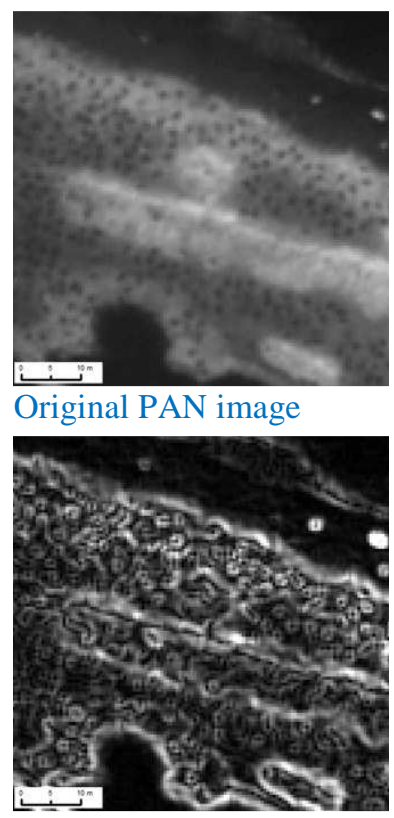

Canny-edge PAN

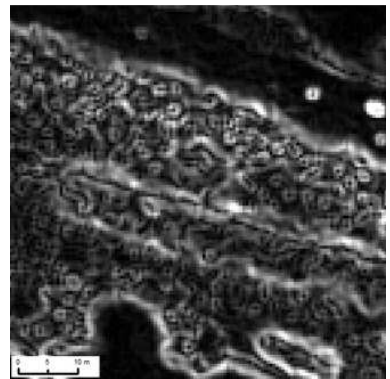

Canny-edge GSM

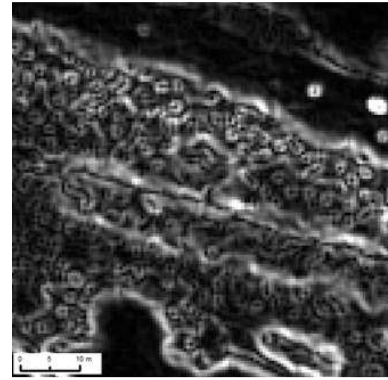

Canny-edge PCA

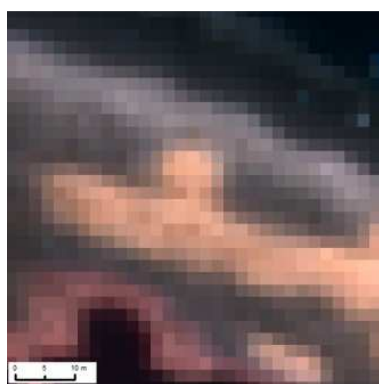

Original MS image

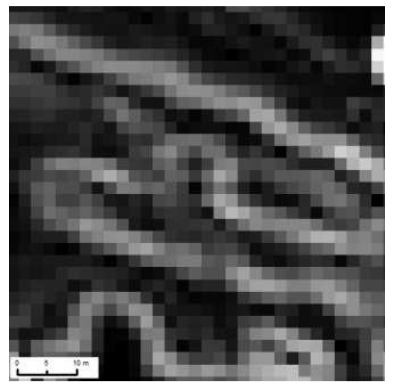

Canny-edge MS

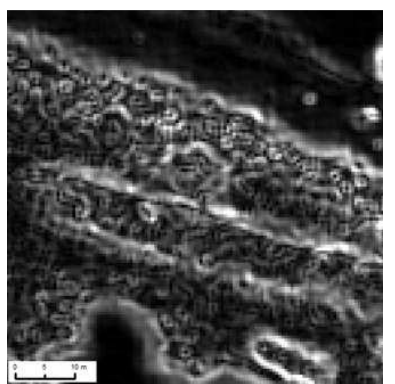

Canny-edge HCS

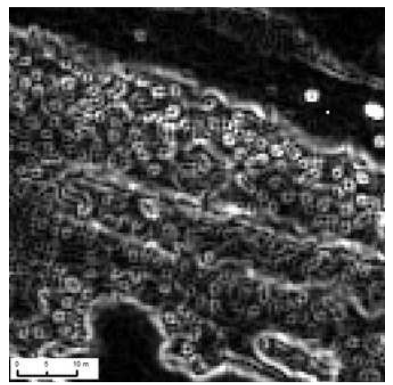

Canny-edge UNB

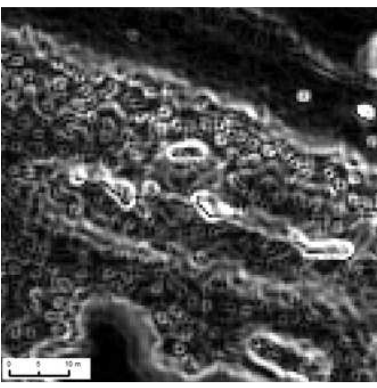

Canny-edge EHL

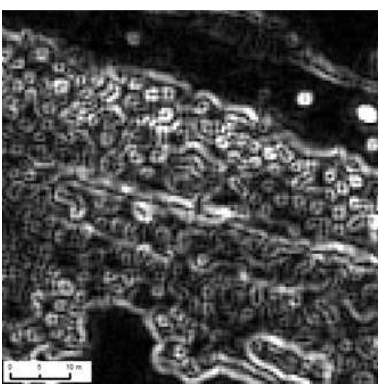

Canny-edge HPF

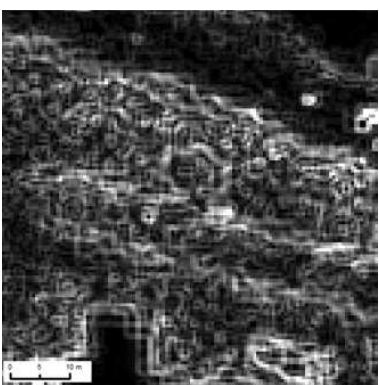

Canny-edge WVL

Figure 9. Zoomed-in views showing Canny-edge filtered images of the original WV-2 PAN and MS images and the fused products. 


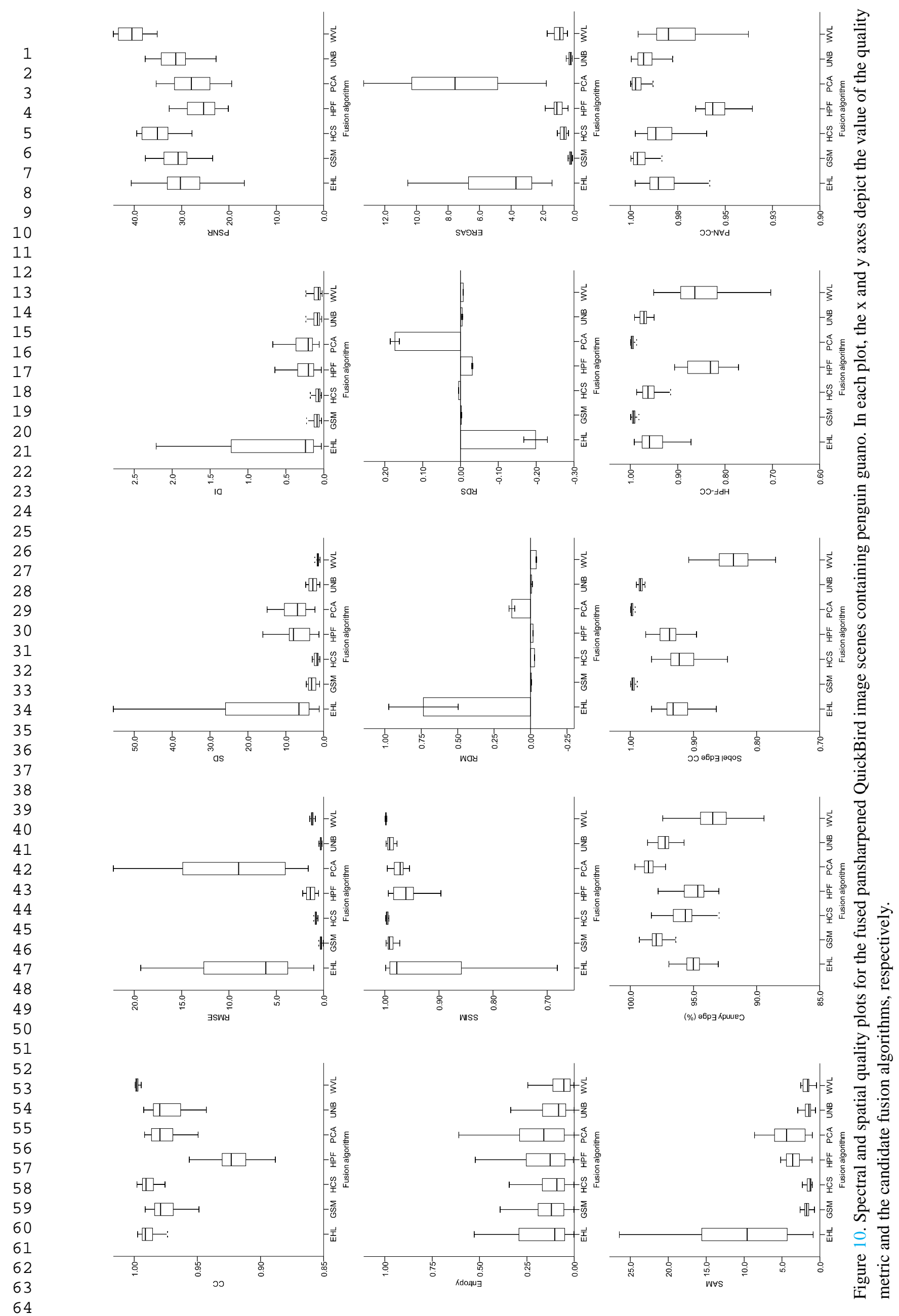




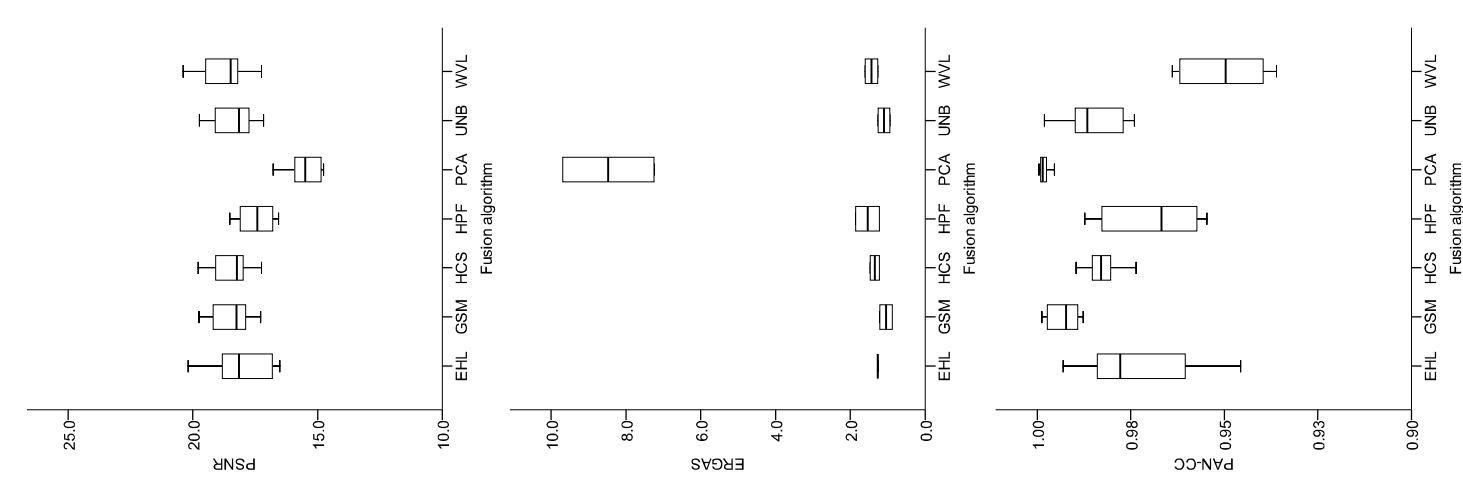

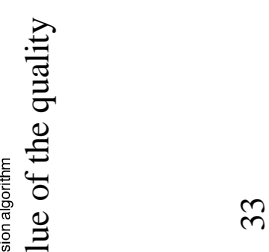
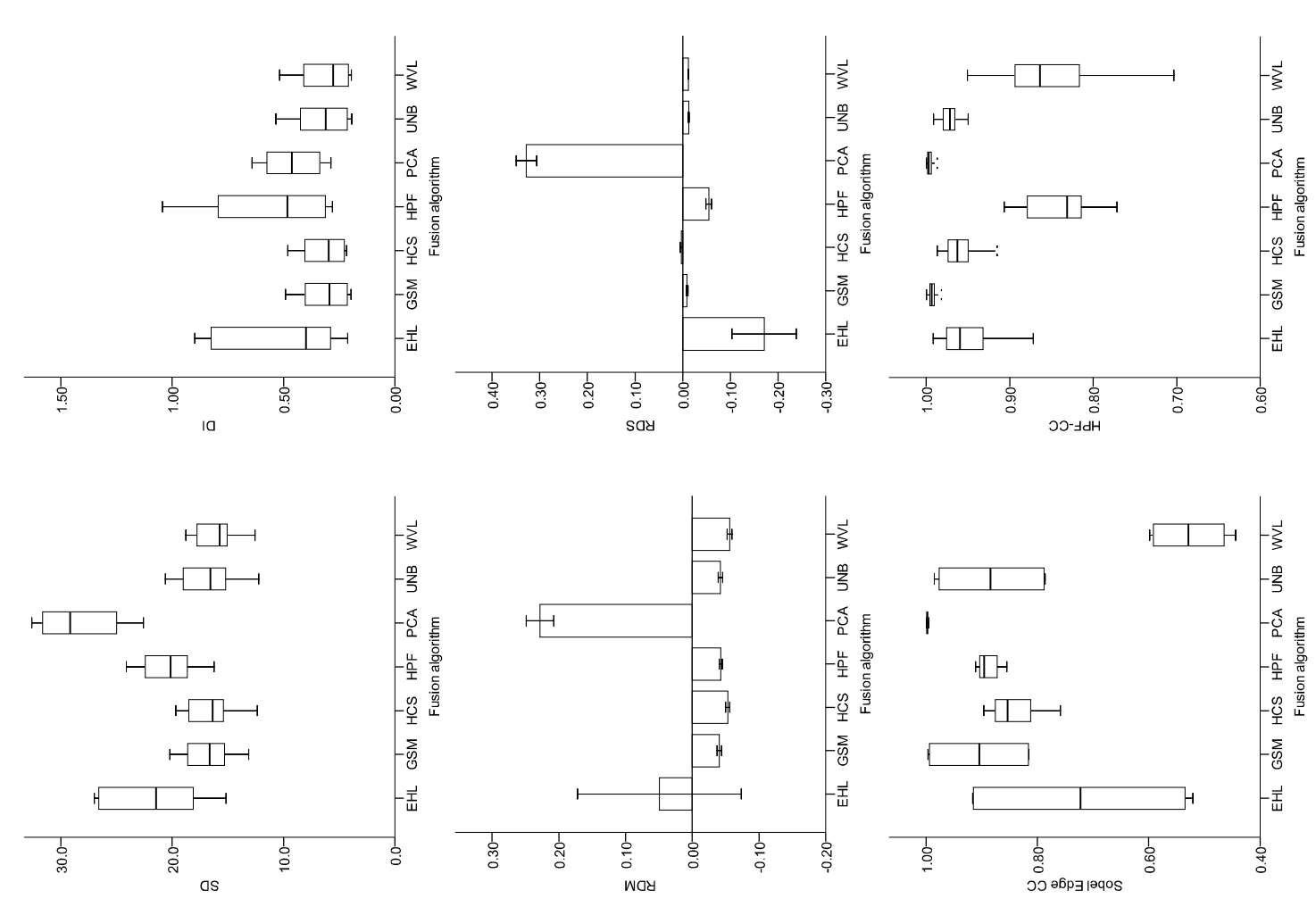

 

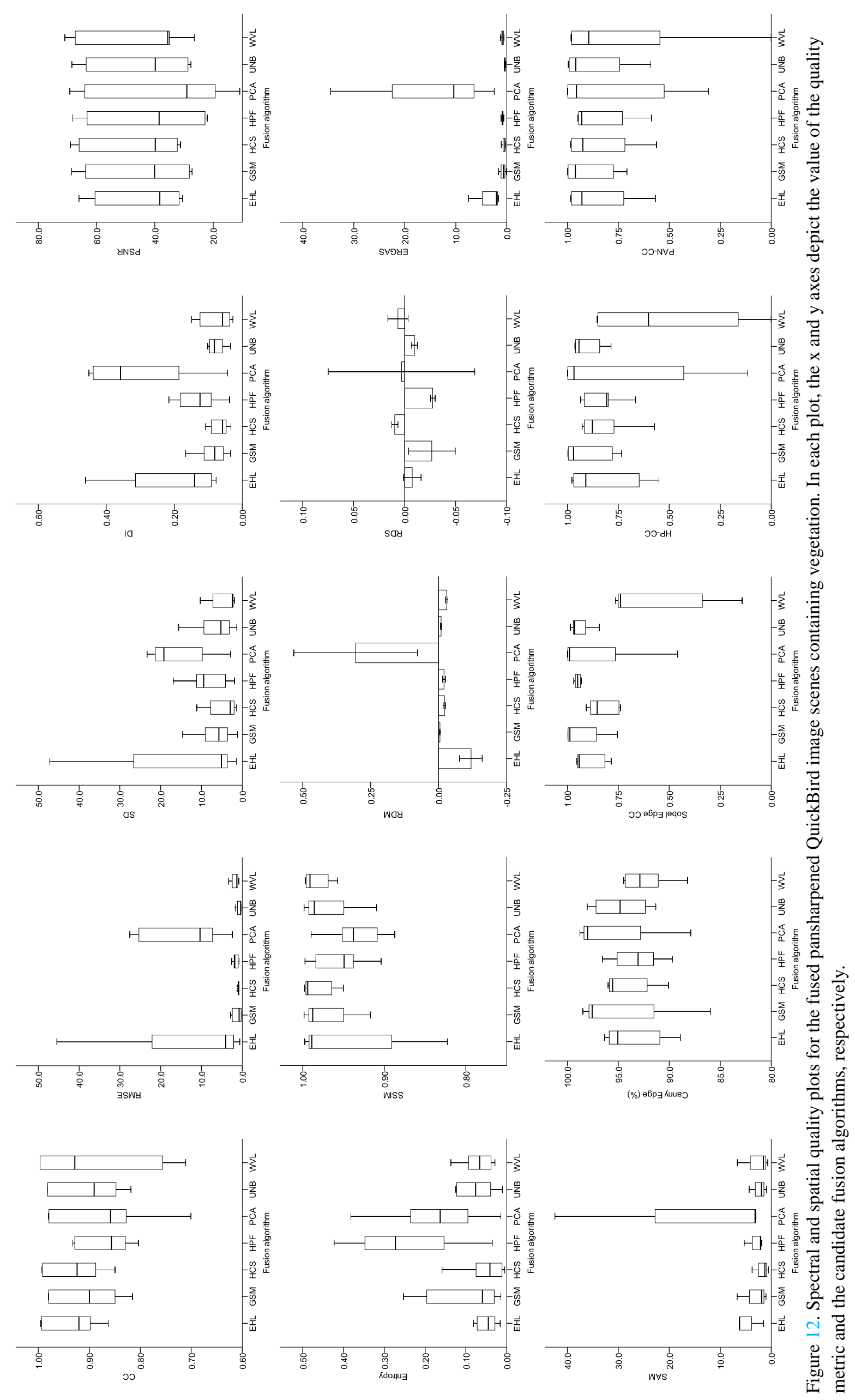

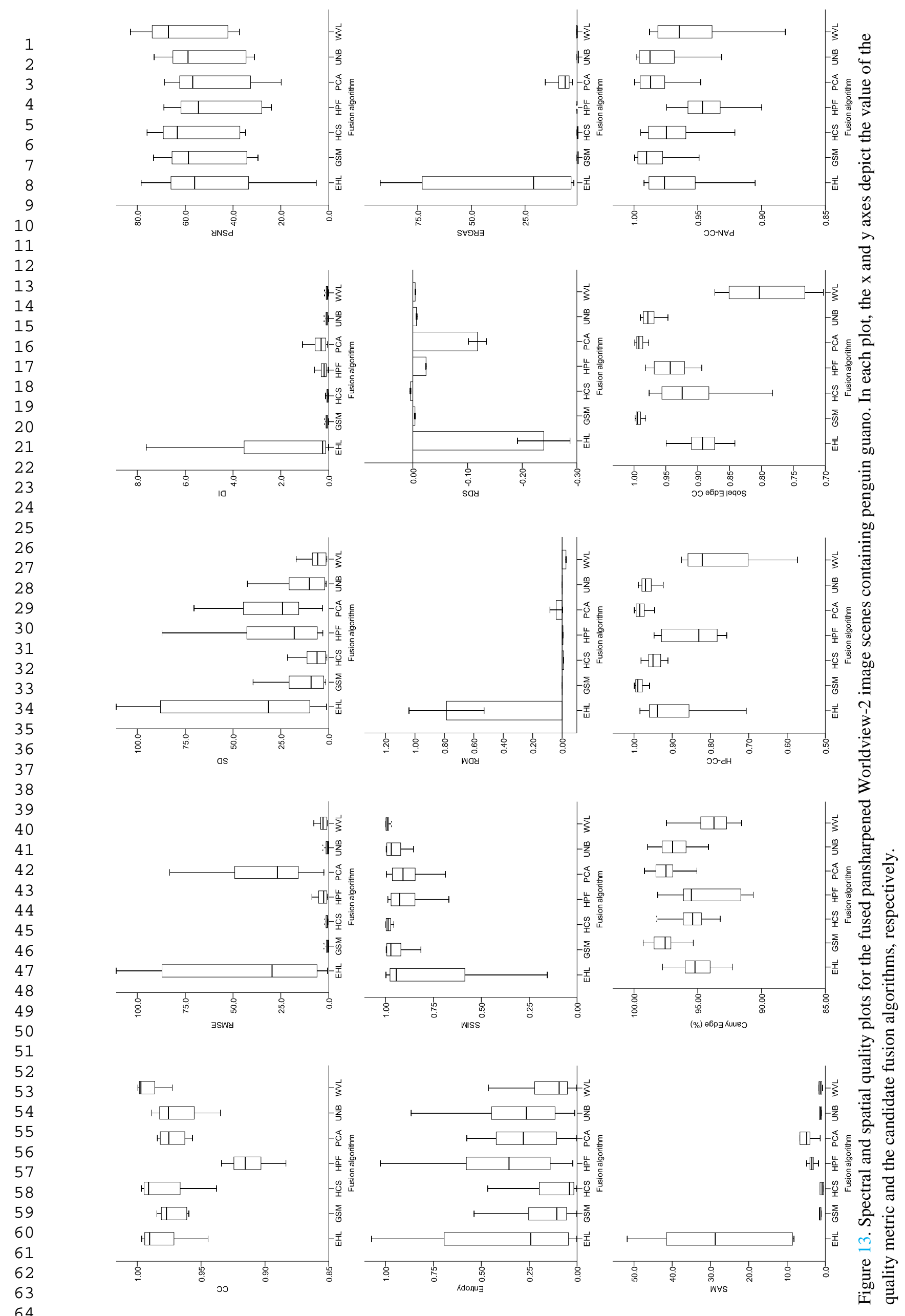


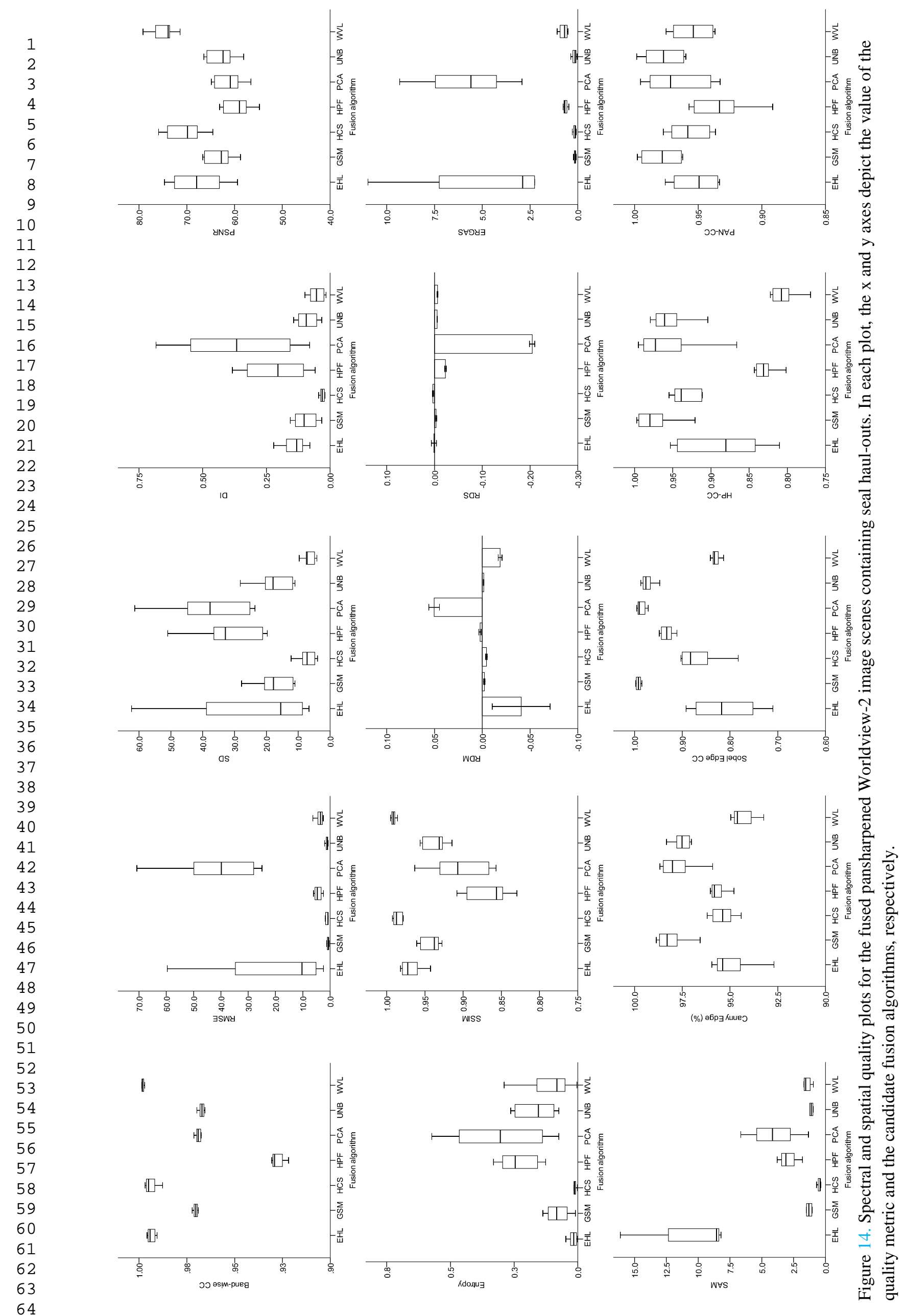



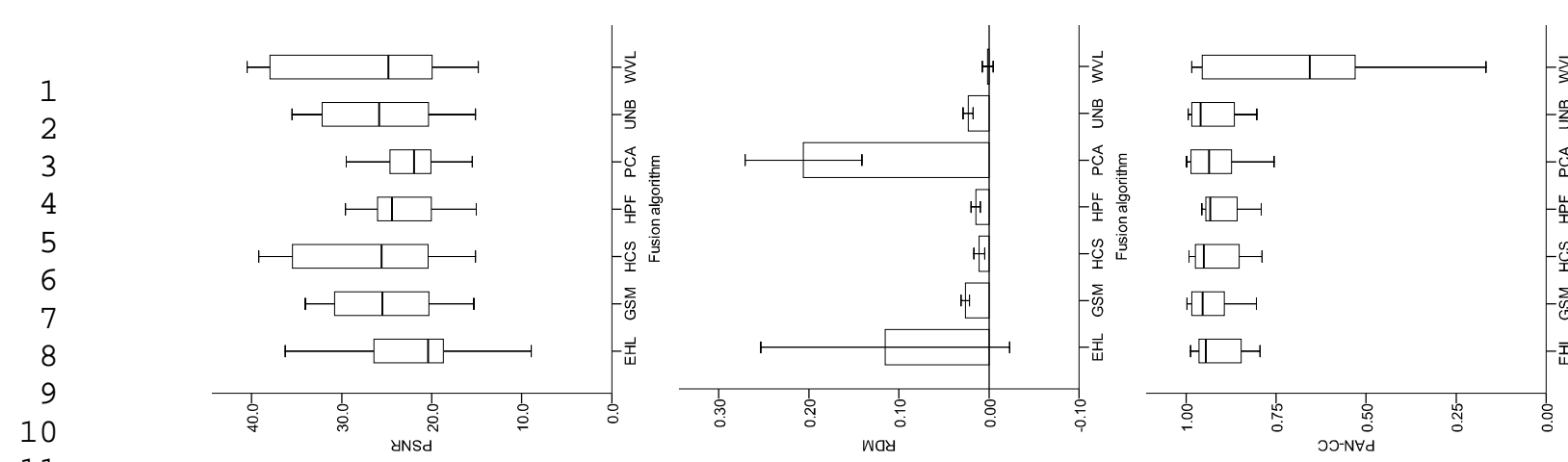

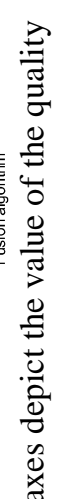




\section{List of Tables}

Table 1. Basic specifications of QuickBird-2 and Worldview-2 sensors.

\begin{tabular}{|c|c|c|c|}
\hline Sensor & Spectral band & Spectral range (nm) & $\begin{array}{l}\text { Spatial resolution (m) } \\
\text { (nadir) }\end{array}$ \\
\hline \multirow{5}{*}{ 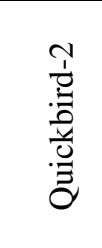 } & Panchromatic & $405-1053$ & 0.61 \\
\hline & Blue & $430-545$ & 2.44 \\
\hline & Green & $466-620$ & 2.44 \\
\hline & Red & $590-710$ & 2.44 \\
\hline & NIR & $715-918$ & 2.44 \\
\hline \multirow{9}{*}{ 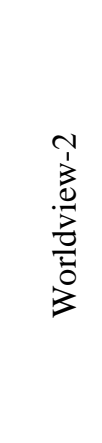 } & Panchromatic & $450-800$ & 0.46 \\
\hline & Coastal & $400-450$ & 1.85 \\
\hline & Blue & $450-510$ & 1.85 \\
\hline & Green & $510-580$ & 1.85 \\
\hline & Yellow & $585-625$ & 1.85 \\
\hline & Red & $630-690$ & 1.85 \\
\hline & Red edge & $705-745$ & 1.85 \\
\hline & NIR1 & $770-895$ & 1.85 \\
\hline & NIR2 & $860-1040$ & 1.85 \\
\hline
\end{tabular}


Table 2. Candidate fusion methods, related literature, and implementation

\begin{tabular}{|c|c|c|}
\hline Algorithm & Reference & Implementation \\
\hline Ehlers fusion (EHL) & $\begin{array}{l}\text { Ling et al., 2007; Ehlers et al., } \\
\text { 2010; Yuhendra, 2012; Jawak and } \\
\text { Luis, 2013; Witharana et al., 2013; } \\
\text { Ehlers and Klonus, } 2014\end{array}$ & ERDAS Imagine \\
\hline $\begin{array}{l}\text { Gram-Schmidt fusion } \\
\text { (GSM) }\end{array}$ & $\begin{array}{l}\text { Laben et al. 2000; Aiazzi et al., } \\
\text { 2007; Karathanassi et al., 2007; } \\
\text { Klonus and Ehlers, 2007; } \\
\text { Yuhendra, 2012; Jawak and Luis, } \\
2013\end{array}$ & ENVI \\
\hline $\begin{array}{l}\text { Hyperspherical color space } \\
\text { (HCS) }\end{array}$ & Padwick et al., 2010 & ERDAS Imagine \\
\hline High-pass filter (HPF) & $\begin{array}{l}\text { Chavez et al.,1991; Gangkofner et } \\
\text { al., 2008; Yang et al., 2012; } \\
\text { Yuhendra, 2012; Jawak and Luis, } \\
\text { 2013; Witharana et al., } 2013\end{array}$ & ERDAS Imagine \\
\hline $\begin{array}{l}\text { Principal component } \\
\text { analysis (PCA) }\end{array}$ & $\begin{array}{l}\text { Chavez et al., 1991; Pohl and van } \\
\text { Genderen, 1998; Kalpoma and } \\
\text { Kudoh, 2007; Karathanassi et al., } \\
\text { 2007; Ling et al., 2008; Yang et } \\
\text { al., 2012; Witharana et al., } 2013\end{array}$ & ERDAS Imagine \\
\hline $\begin{array}{l}\text { University of New } \\
\text { Brunswick (UNB) }\end{array}$ & $\begin{array}{l}\text { Zhang, 2002; Zhang, 2004; } \\
\text { Alparone et al., 2007; Karathanassi } \\
\text { et al., 2007; Wong et al., 2011; } \\
\text { Zhan and Jixian, } 2014\end{array}$ & PCI Geomatics \\
\hline Wavelet Transform (WVL) & $\begin{array}{l}\text { Vijayaraj et al., 2006; Karathanassi } \\
\text { et al., 2007; Gangkofner et al., } \\
\text { 2008; Yakhdani and Azizi, 2010; } \\
\text { Yang et al., 2012; Yuhendra, 2012; } \\
\text { Jawak and Luis, } 2013\end{array}$ & ERDAS Imagine \\
\hline
\end{tabular}




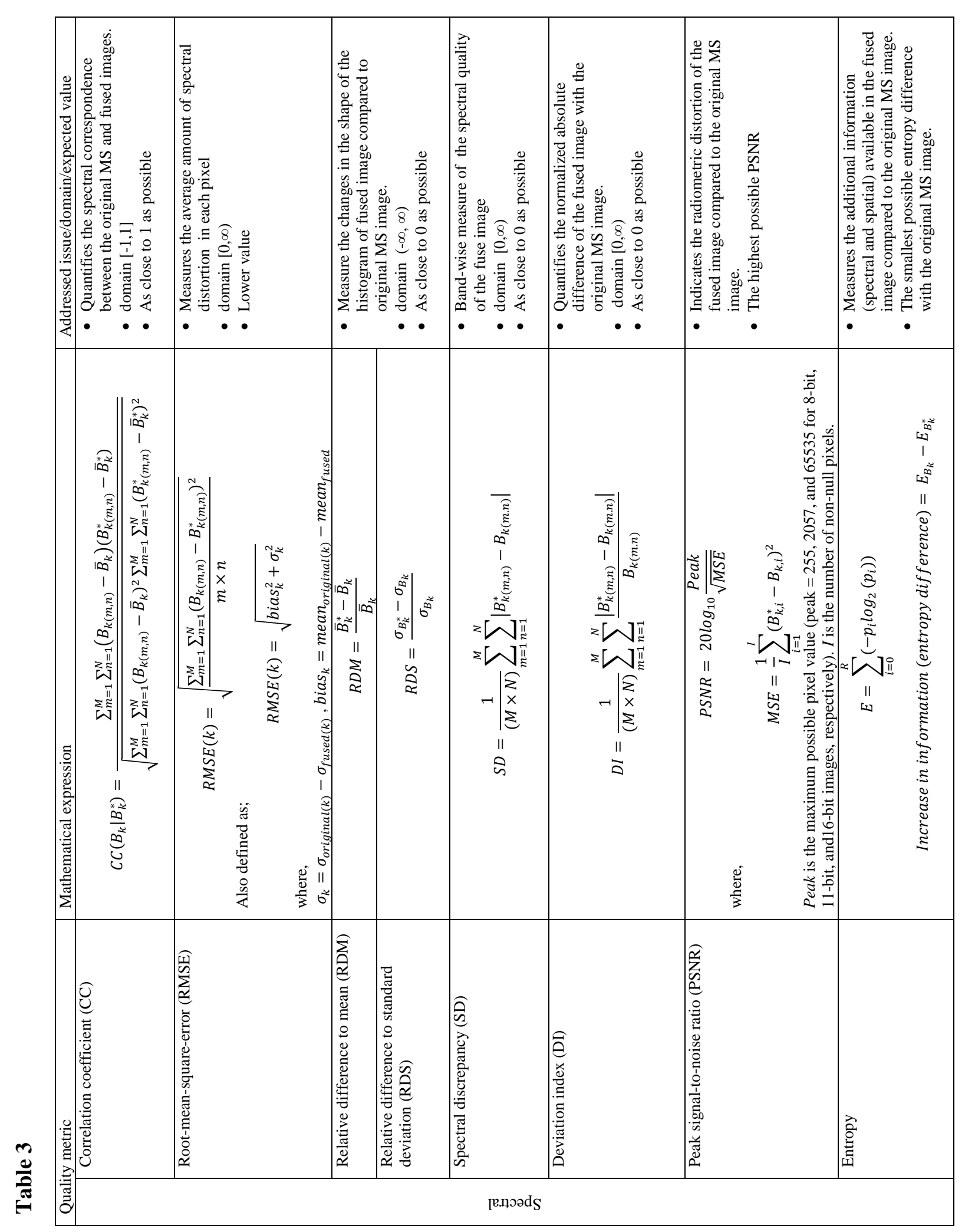




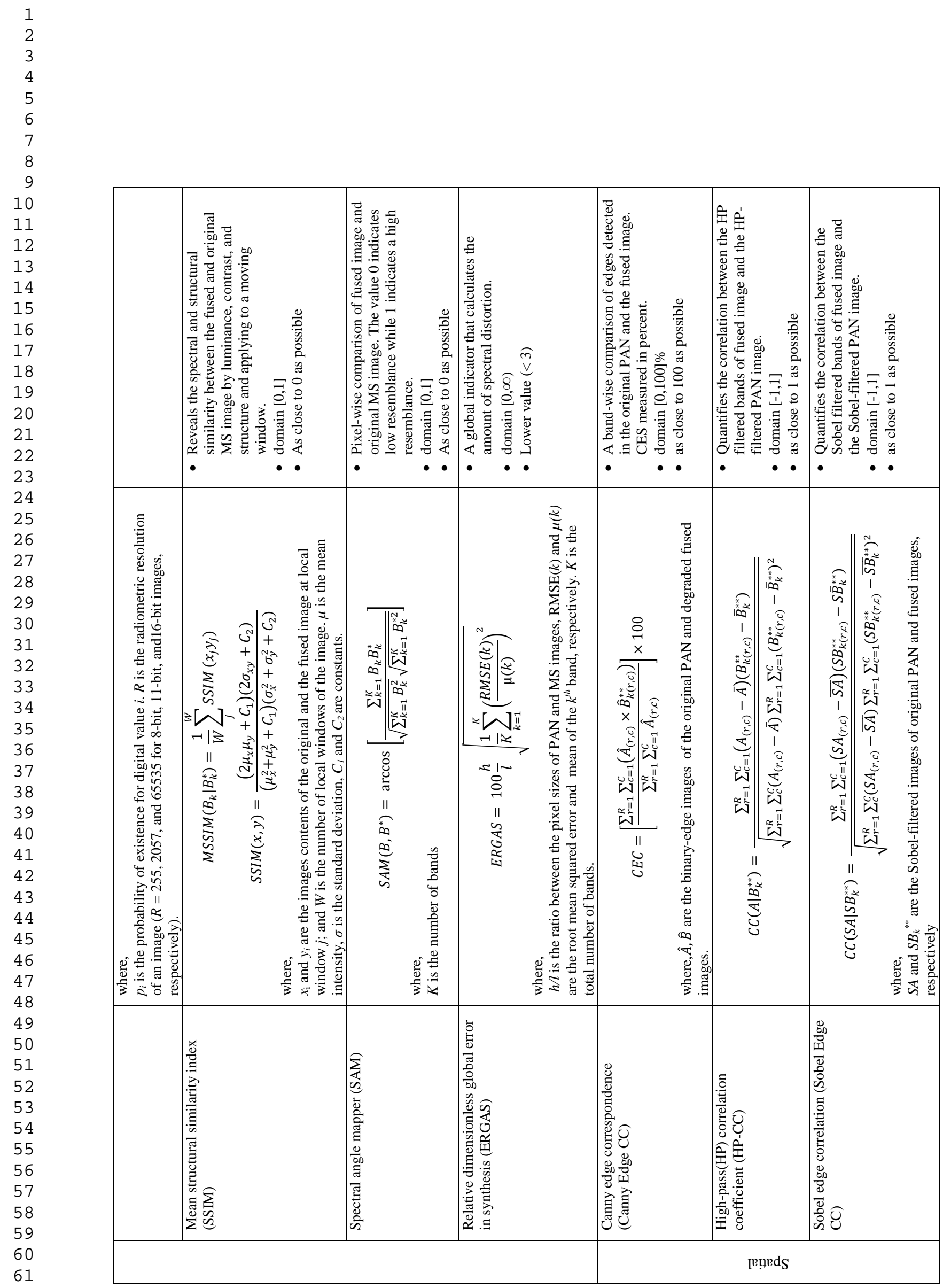




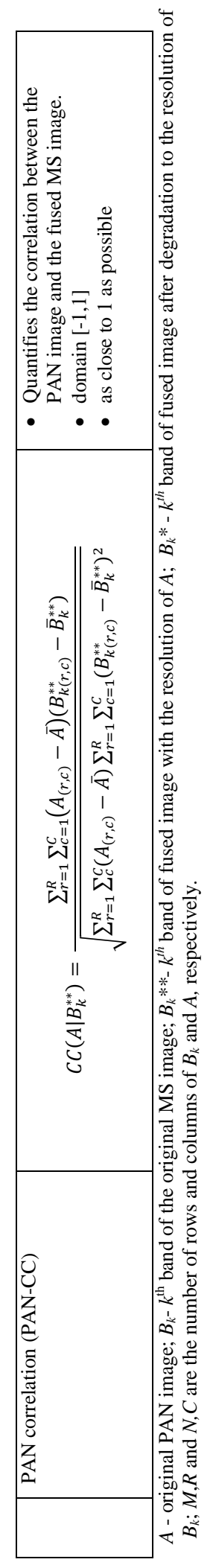

62

63

64

65 
Table. 4

\begin{tabular}{|c|c|c|c|c|c|}
\hline \multirow{2}{*}{$\begin{array}{l}\text { Expert } \\
\text { focus }\end{array}$} & \multirow{2}{*}{$\begin{array}{l}\text { Target } \\
\text { features }\end{array}$} & \multicolumn{4}{|c|}{ Fusion quality } \\
\hline & & \multicolumn{2}{|c|}{ Best } & \multicolumn{2}{|c|}{ Worst } \\
\hline \multirow{4}{*}{ 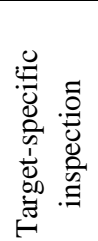 } & \multirow{4}{*}{$\begin{array}{l}\text { Guano } \\
\text { Seals } \\
\text { Vegetation }\end{array}$} & Analyst 1 & Analyst 2 & Analyst 1 & Analyst 2 \\
\hline & & HPF,UNB & GSM,UNB & EHL,WVL & EHL,WVL \\
\hline & & HPF,UNB & HPF,UNB & EHL,WVL & EHL,WVL \\
\hline & & HPF,UNB & HPF,UNB & EHL,WVL & EHL,WVL \\
\hline \multirow{4}{*}{ 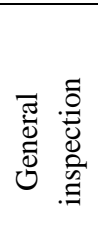 } & \multirow{4}{*}{$\begin{array}{l}\text { Guano } \\
\text { Seals } \\
\text { Vegetation }\end{array}$} & Analyst 3 & Analyst 4 & Analyst 3 & Analyst 4 \\
\hline & & HPF,UNB & HPF,UNB & EHL,WVL & EHL,WVL \\
\hline & & HPF,UNB & HPF,UNB & EHL,WVL & EHL,WVL \\
\hline & & HPF,UNB & HPF,UNB & EHL,WVL & EHL,WVL \\
\hline
\end{tabular}




\section{Literature Cited}

Abileah, R., 2002. Marine mammal census using space satellite imagery. U.S. Navy Journal of Underwater Acoustics 52 (3) July.

Ainley, D.G., LaRue, M.A., Stammerjohn, S.E., Stirling, I., Siniff, D.B., 2015. Apparent decline of Weddell seal numbers along the Northern Victoria Land Coast. Marine Mammal Science, DOI: 10.1111/mms.12220.

Aksoy, S. and Cinbis, R.G., 2010. Image Mining Using Directional Spatial Constraints. Geoscience and Remote Sensing Letters, IEEE, 7: 33-37.

Alparone, L., Wald, L., Chanussot, J., Thomas, C., Gamba, P. and Bruce, L. M., 2007. Comparison of Pansharpening Algorithms: Outcome of the 2006 GRS-S Data-Fusion Contest. IEEE Transactions on Geoscience and Remote Sensing, 45(10): 3012-3021.

Alparone, L., Aiazzi, B., Baronti, S., Garzelli, A. and Nencini, F., 2006. Information-theoretic image fusion assessment without reference, Image Information Mining for Security and Intelligence. ESA-EUSC 2006.

Alparone, L., Baronti, S., Garzelli, A. and Nencini, F., 2004. A global quality measurement of pan-sharpened multispectral imagery. IEEE Geoscience and Remote Sensing Letters, 1(4): 313-317.

Ardila, J.P., Bijker, W., Tolpekin, V.A. and Stein, A., 2012. Context-sensitive extraction of tree crown objects in urban areas using VHR satellite images. International Journal of Applied Earth Observation and Geoinformation, 15(0): 57-69.

Ashraf, S., Brabyn, L. and Hicks, B.J., 2012. Image data fusion for the remote sensing of freshwater environments. Applied Geography, 32(2): 619-628.

Barber-Meyer, S., Kooyman, G. and Ponganis, P., 2007. Estimating the relative abundance of emperor penguins at inaccessible colonies using satellite imagery. Polar Biology, 30(12): 1565-1570.

Beguet, B., Guyon, D., Boukir, S. and Chehata, N., 2014. Automated retrieval of forest structure variables based on multi-scale texture analysis of VHR satellite imagery. ISPRS Journal of Photogrammetry and Remote Sensing, 96: 164-178.

Blaschke, T., Hay,G. J., Kelly, M., Lang, S., Hofmann, P., Addink, E., Queiroz Feitosa, R., van der Meer, F., van der Werff, H., van Coillie, F., and Tiede, D. 2014. Geographic Object-Based Image Analysis -Towards a new paradigm. ISPRS Journal of Photogrammetry and Remote Sensing, 87(0): 180-191.

Chavez, P.S., Sides, S.C. and Anderson, J.A., 1991. Comparison of three different methods to merge multiresolution and multispectral data: Landsat TM and SPOT panchromatic. Photogrammetric Engineering \& Remote Sensing, 57(3): 295-303.

Civco, D., Chabaeva, A., and Parent, J. 2009. KH-series satellite imagery and Landsat MSS data fusion in support of assessing urban land use growth. In: M. Ulrich and L.C. Daniel (Editors). SPIE, pp. 747-80I.

De Bethune, S., Muller, F. and Donnay, J.P., 1998. Fusion of multispectral and panchromatic images by local mean and variance matching filtering techniques, Fusion of Earth Data. IRS-1D Users Handbook Hyerabad: National Remote Sensing Agency, Sophia Antipolis, Nice, France, pp. 31-37.

Ehlers, M., Klonus, S., Johan Ã., Strand, P.r. and Rosso, P., 2010. Multi-sensor image fusion for pansharpening in remote sensing. International Journal of Image and Data Fusion, 1(1): 25-45.

Ehlers, M. and Klonus, S., 2014. Scale Issues In Multisensor Image Fusion, Scale Issues in Remote Sensing. John Wiley \& Sons, Inc., pp. 11-33. 
ERDAS, 2014. ERDAS IMAGINE Field Guide.

Fretwell, P.T. and Trathan, P.N., 2009. Penguins from space: faecal stains reveal the location of emperor penguin colonies. Global Ecology and Biogeography, 18(5): 543-552.

Fretwell, P.T., Convey, P., Fleming, A.H., Peat, H.J. and Hughes, K.A., 2011. Detecting and mapping vegetation distribution on the Antarctic Peninsula from remote sensing data. Polar Biology, 34(2): 273-281.

Fretwell, P. T., LaRue, M. A., Morin, P., Kooyman, G. L., Wienecke, B., Ratcliffe, N., Fox, A. J., Fleming, A. H., Porter, C. and Trathan, P. N., 2012. An Emperor Penguin Population Estimate: The First Global, Synoptic Survey of a Species from Space. PLoS ONE, 7: e33751.

Fretwell, P.T., Phillips, R.A., Brooke, M.D.L., Fleming, A.H. and McArthur, A., 2015. Using the unique spectral signature of guano to identify unknown seabird colonies. Remote Sensing of Environment, 156(0): 448456.

Fretwell, P.T., Staniland, I.J. and Forcada, J., 2014. Whales from Space: Counting Southern Right Whales by Satellite. PLoS ONE, 9(2): e88655.

Gamba, P., 2014. Image and data fusion in remote sensing of urban areas: status issues and research trends. International Journal of Image and Data Fusion, 5(1): 2-12.

Gangkofner, U.G., Pradhan, P. S. and Holcomb, D.W., 2008. Optimizing the High-Pass Filter Addition Technique for Image Fusion. Photogrammetric Engineering \& Remote Sensing, 74(9): 1107-1118.

Garcia, J.A., Rodriguez-Sánchez, R., Fdez-Valdivia, J. and Toet, A., 2012. Visual efficiency of image fusion methods. International Journal of Image \& Data Fusion, 3(1): 39-69.

Guo, Q., Chen, S., Leung, H. and Liu, S., 2010. Covariance intersection based image fusion technique with application to pansharpening in remote sensing. Information Sciences, 180(18): 3434-3443.

Hallada, W.A. and Cox, S., 1983. Image sharpening for mixed spatial and spectral resolution satellite systems, 17th International Symposium on Remote Sensing of Environment, Ann Arbor, Michigan, pp. 1023-1032.

Huang, X., Lu, Q. and Zhang, L., 2014. A multi-index learning approach for classification of high-resolution remotely sensed images over urban areas. ISPRS Journal of Photogrammetry and Remote Sensing, 90(0): $36-48$.

Jawak, S.D. and Luis, A.J., 2012. A spectral index ratio-based Antarctic land-cover mapping using hyperspatial 8band Worldview-2 imagery. Polar Science, 7(1): 18-38.

Jawak, S.D. and Luis, A.J., 2014. A Semiautomatic Extraction of Antarctic Lake Features Using Worldview-2 Imagery. Photogrammetric Engineering \& Remote Sensing, 80(10): 33-46.

Karathanassi, V., Kolokousis, P. and Ioannidou, S., 2007. A comparison study on fusion methods using evaluation indicators. International Journal of Remote Sensing, 28(10): 2309-2341.

Karlson, M., Reese, H. and Ostwald, M., 2014. Tree Crown Mapping in Managed Woodlands (Parklands) of SemiArid West Africa Using Worldview-2 Imagery and Geographic Object Based Image Analysis. Sensors, 14(12): 22643-22669.

Kim, M., Holt, J.B. and Madden, M., 2011. Comparison of Global- and Local-scale Pansharpening for Rapid Assessment of Humanitarian Emergencies. Photogrammetric Engineering \& Remote Sensing, 77(1): 51-63.

Klonus, S. and Ehlers, M., 2007. Image Fusion Using the Ehlers Spectral Characteristics Preservation Algorithm. GIScience \& Remote Sensing, 44(2): 93-116. 
Laben, C.A., Bernard, V., and Brower, W., 2000. Process for enhancing the spatial resolution of multispectral imagery using pan-sharpening. United States Patent Application No. 6,011,875.

LaRue, M.A., Rotella, J., Garrott, R., Siniff , D., Ainley, D., Stauffer, G., Porter, C., and Morin, P., 2011. Satellite imagery can be used to detect variation in abundance of Weddell seals (Leptonychotes weddellii) in Erebus Bay, Antarctica. Polar Biology, 34(11): 1727-1737.

LaRue, M.A., 2014. Using high-resolution satellite imagery to assess populations of animals in Antarctica, Ph.D. Dissertation, University of Minnesota, Minneapolis, http://hdl.handle.net/11299/163250.

LaRue, M. A., Lynch, H. J., Lyver, P. O. B., Barton, K., Ainley, D. G., Pollard , A., Fraser, W. R., and Ballard G., 2014. A method for estimating colony sizes of Adélie penguins using remote sensing imagery. Polar Biology, 37(4): 507-517.

Li, S., Li, Z. and Gong, J., 2010. Multivariate statistical analysis of measures for assessing the quality of image fusion. International Journal of Image and Data Fusion, 1(1): 47-66.

Ling, Y., Ehlers, M., Usery, E.L. and Madden, M., 2007. FFT-enhanced IHS transform method for fusing highresolution satellite images. ISPRS Journal of Photogrammetry and Remote Sensing, 61(6): 381-392.

Ling, Y., Ehlers, M., Usery, E.L. and Madden, M., 2008. Effects of spatial resolution ratio in image fusion. International Journal of Remote Sensing, 29(7): 2157-2167.

Liu, J., Li, P. and Wang, X., 2015. A new segmentation method for very high resolution imagery using spectral and morphological information. ISPRS Journal of Photogrammetry and Remote Sensing, 101(0): 145-162.

Liu, Q., Wang, Y. and Zhang, Z., 2014. Pan-sharpening based on geometric clustered neighbor embedding. Optical Engineering, 53(9): 093109-1 - 093109-16.

Lynch, H.J. and LaRue, M.A., 2014. First global census of the Adélie Penguin. The Auk, 131(4): 457-466.

Lynch, H.J., White, R., Black, A.D. and Naveen, R., 2012. Detection, differentiation, and abundance estimation of penguin species by high-resolution satellite imagery. Polar Biology, 35(6): 963-968.

Makarau, A., Palubinskas, G. and Reinartz, P., 2012. Analysis and selection of pan-sharpening assessment measures. Journal of Applied Remote Sensing, 6.

McMahon, C. R., Howe, H., van den Hoff, J., Alderman, R., Brolsma, H. and Hindell, M. A., 2014. Satellites, the All-Seeing Eyes in the Sky: Counting Elephant Seals from Space. PLoS ONE, 9(3): e92613.

Myint, S.W., Gober, P., Brazel, A., Grossman-Clarke, S. and Weng, Q., 2011. Per-pixel vs. object-based classification of urban land cover extraction using high spatial resolution imagery. Remote Sensing of Environment, 115(5): 1145-1161.

Nikolakopoulos, K.G., 2008. Comparison of nine fusion techniques for very high resolution data. Comparison of nine fusion techniques for very high resolution data, 74(5): 647-660.

Padwick C., Deskevich M., Pacifici F., Smallwood S. (2010) - Worldview-2 pan-sharpening. ASPRS 2010 Annual Conference, San Diego, California, pp.14.

Pohl, C. and Van Genderen, J.L., 1998. Multisensor image fusion in remote sensing: concepts, methods and applications. International Journal of Remote Sensing, 19(5): 823-854.

Pohl, C. and van Genderen, J., 2014. Remote sensing image fusion: an update in the context of Digital Earth. International Journal of Digital Earth, 7(2): 158-172. 
Pradhan, P.S., King, R.L., Younan, N.H. and Holcomb, D.W., 2006. Estimation of the Number of Decomposition Levels for a Wavelet-Based Multiresolution Multisensor Image Fusion. IEEE Transactions on Geoscience and Remote Sensing, 44(12): 3674-3686.

Ranchin, T. and Wald, L., 2000. Fusion of high spatial and spectral resolution images: The ARSIS concept and its implementation. Photogrammetric Engineering \& Remote Sensing, 66(1): 49-61.

Ranchin, T., Aiazzi, B., Alparone, L., Baronti, S. and Wald, L., 2003. Image fusion--the ARSIS concept and some successful implementation schemes. ISPRS Journal of Photogrammetry and Remote Sensing, 58(1-2): 418.

Samadzadegan, F., Fathollahi, F. and Rezaee, M., Comparing different IHS-based pan-sharpening techniques for worldview-2 high resolution satellite imagery, Geoscience and Remote Sensing Symposium (IGARSS), 2012 IEEE International, pp. 2296-2299.

Schwaller, M.R., Benninghoff, W.S. and Olson, C.E., 1984. Prospects for satellite remote sensing of Adelie penguin rookeries. International Journal of Remote Sensing, 5(5): 849-853.

Schwaller, M.R., Olson Jr, C.E., Ma, Z., Zhu, Z. and Dahmer, P., 1989. A remote sensing analysis of Adélie penguin rookeries. Remote Sensing of Environment, 28(0): 199-206.

Schwaller, M.R., Southwell, C.J. and Emmerson, L.M., 2013. Continental-scale mapping of Adélie penguin colonies from Landsat imagery. Remote Sensing of Environment, 139(0): 353-364.

Vijayaraj, V., Nicolas, H.Y. and Charles, G.O.H., 2006. Quantitative analysis of pansharpened images. Optical Engineering, 45(4): 046202.

Wald, L. and Ranchin, T., 1997. Fusion of satellite images of different spatial resolutions: Assessing the quality of resulting images. Photogrammetric Engineering \& Remote Sensing, 63(6): 691-699.

Wald, L., 2000. Quality of high resolution synthesised images: Is there a simple criterion?. In: T. Ranchin and L. Wald (Editors), Fusion of Earth data: merging point measurements, raster maps and remotely sensed images. SEE/URISCA,Nice, France, Sophia Antipolis, France, pp. 166.

Wald, L., 2002. Data Fusion: Definitions and Architectures - Fusion of Images of Different Spatial Resolutions. Les Presses, Ecole des Mines de Paris,, Paris, France, 200 pp.

Wang, Z. and Bovik, A.C., 2002. A universal image quality index. IEEE Signal Processing Letters, 9: 81-84.

Wang, Z., Bovik, A.C., Sheikh, H. and Simoncelli, E.P., 2004. Image quality assessment: from error visibility to structural similarity. IEEE Transactions on Image Processing, 13(1): 600-612.

Weidner, U., 2010. Pansharpening - relating quantitative quality measures to impact on results of subsequent processing steps. In: W. W. and B. Székely (Editors), ISPRS TC VII Symposium - 100 Years ISPRS. IAPRS, Vienna, Austria.

Witharana, C., 2012. Who Does What Where? Advanced Earth Observation for Humanitarian Crisis Management, Proceedings of the 6th International Conference on Information and Automation. Beijing, China. IEEE paper no. ICIAfS'12 1569613211.

Witharana, C. and Civco, D.L., 2012. Evaluating remote sensing image fusion algorithms for use in humanitarian crisis management. SPIE Remote Sensing, Edinburg, United Kingdom, Paper no. 8538-5. 
Witharana, C., Nishshanka, U. and Gunatilaka. J., 2013a. Remote Sensing of Ecological Hotspots: Value-added Information from Multiple Data Sources for Horton Plains National Park, Sri Lanka, Journal of Geological Society of Sri Lanka, 15,147-162.

Witharana, C., Civco, D.L. and Meyer, T.H., 2013b. Evaluation of pansharpening algorithms in support of earth observation based rapid-mapping workflows. Applied Geography, 37(0): 63-87.

Witharana, C. and Civco, D.L., 2014. Optimizing multi-resolution segmentation scale using empirical methods: Exploring the sensitivity of the supervised discrepancy measure Euclidean distance 2 (ED2). ISPRS Journal of Photogrammetry and Remote Sensing, 87: 108-121.

Witharana, C., Civco, D.L. and Meyer, T.H., 2014. Evaluation of data fusion and image segmentation in earth observation based rapid mapping workflows. ISPRS Journal of Photogrammetry and Remote Sensing, 87(0): 1-18.

Xu, Q., Zhang, Y. and Li, B., 2014. Recent advances in pansharpening and key problems in applications. International Journal of Image and Data Fusion, 5(3): 175-195.

Xydeas, C. and Petrovic, V., 2000. Objective image fusion performance measure. Electronic Letters, 36(4): 308-309.

Yakhdani, M.F. and Azizi, A., 2010. Quality assessment of image fusion techniques for multisensor high resolution satellite images (case study: IRS-p5 and IRS-p6 satellite images). In: W. W. and B. Székely (Editors), ISPRS TC VII Symposium - 100 Years ISPRS. IAPRS, Vienna, Austria, pp. Part 7B.

Yang, Z., Wang, T., Skidmore, A. K., de Leeuw, J., Said, M. Y., and Freer, J., 2014. Spotting East African Mammals in Open Savannah from Space. PLoS ONE, 9(12): e115989.

Yuhendra, Alimuddin, I., Sumantyo, J.T.S. and Kuze, H., 2012. Assessment of pan-sharpening methods applied to image fusion of remotely sensed multi-band data. International Journal of Applied Earth Observation and Geoinformation, 18(0): 165-175.

Zhang, Y., 2002. A new automatic approach for effectively fusing Landsat as well as IKONOS images. IEEE Transactions on Geoscience and Remote Sensing: 2429-2431.

Zhang, J., 2014. Progress in Immage and Data Fusion: The road ahead. International Journal of Image \& Data Fusion, 3(1): 1-2. 